Published in final edited form as:

Nature. 2014 March 27; 507(7493): 455-461. doi:10.1038/nature12787.

\title{
An atlas of active enhancers across human cell types and tissues
}

Robin Andersson ${ }^{\# 1}$, Claudia Gebhard\#2, Irene Miguel-Escalada ${ }^{3}$, Ilka Hoof ${ }^{1}$, Jette Bornholdt ${ }^{1}$, Mette Boyd ${ }^{1}$, Yun Chen ${ }^{1}$, Xiaobei Zhao ${ }^{1,4}$, Christian Schmidl ${ }^{2}$, Takahiro Suzuki ${ }^{5,6}$, Evgenia Ntini ${ }^{7}$, Erik Arner ${ }^{5,6}$, Eivind Valen ${ }^{1,8}$, Kang Li ${ }^{1}$, Lucia Schwarzfischer ${ }^{2}$, Dagmar Glatz ${ }^{2}$, Johanna Raithel $^{2}$, Berit Lilje ${ }^{1}$, Nicolas Rapin ${ }^{1,9}$, Frederik Otzen Bagger ${ }^{1,9}$, Mette Jørgensen ${ }^{1}$, Peter Refsing Andersen 7 , Nicolas Bertin ${ }^{5,6}$, Owen Rackham ${ }^{5,6}$, A. Maxwell Burroughs ${ }^{5,6}$, J. Kenneth Baillie $^{10}$, Yuri Ishizu ${ }^{5,6}$, Yuri Shimizu ${ }^{5,6}$, Erina Furuhata ${ }^{5,6}$, Shiori Maeda ${ }^{5,6}$, Yutaka Negishi5,6, Christopher J. Mungall ${ }^{11}$, Terrence F. Meehan ${ }^{12}$, Timo Lassmann ${ }^{5,6}$, Masayoshi Itoh ${ }^{5,6,13}$, Hideya Kawaji ${ }^{5,13}$, Naoto Kondo ${ }^{5,13}$, Jun Kawai ${ }^{5,13}$, Andreas Lennartsson ${ }^{14}$, Carsten O. Daub ${ }^{5,6,14}$, Peter Heutink $^{15}$, David A. Hume ${ }^{10}$, Torben Heick Jensen ${ }^{7}$, Harukazu Suzuki ${ }^{5,6}$, Yoshihide

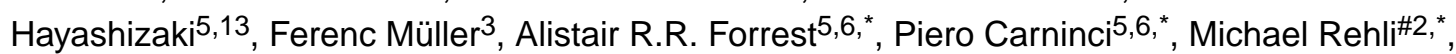
and Albin Sandelin\#1, ${ }^{*}$

${ }^{1}$ The Bioinformatics Centre, Department of Biology \& Biotech Research and Innovation Centre, University of Copenhagen, Ole Maaloes Vej 5, DK-2200 Copenhagen, Denmark ${ }^{2}$ Department of Internal Medicine III, University Hospital Regensburg, Franz-Josef-Strauss-Allee 11, 93042 Regensburg, Germany ${ }^{3}$ School of Clinical and Experimental Medicine, College of Medical and Dental Sciences, University of Birmingham, Edgbaston, Birmingham B15 2TT, UK ${ }^{4}$ Lineberger Comprehensive Cancer Center, University of North Carolina, Chapel Hill, NC 27599, USA ${ }^{5}$ RIKEN OMICS Science Centre, RIKEN Yokohama Institute, 1-7-22 Suehiro-cho, Tsurumi-ku, Yokohama City, Kanagawa, 230-0045, Japan ${ }^{6}$ RIKEN Center for Life Science Technologies (Division of Genomic Technologies), RIKEN Yokohama Institute, 1-7-22 Suehiro-cho, Tsurumi-ku, Yokohama City, Kanagawa, 230-0045, Japan ${ }^{7}$ Centre for mRNP Biogenesis and Metabolism, Department of Molecular Biology and Genetics, C.F. Møllers Alle 3, Bldg. 1130, DK-8000 Aarhus, Denmark ${ }^{8}$ Department of Molecular and Cellular Biology, Harvard University, USA ${ }^{9}$ The Finsen Laboratory, Rigshospitalet and Danish Stem Cell Centre (DanStem), University of Copenhagen, Ole Maaloes Vej 5, DK-2200, Denmark ${ }^{10}$ Roslin Institute, Edinburgh University, Easter Bush, Midlothian, EH25 9RG Scotland, UK ${ }^{11}$ Genomics Division, Lawrence Berkeley National Laboratory, 1 Cyclotron Road MS 64-121, Berkeley, CA 94720, USA ${ }^{12}$ EMBL Outstation -

\footnotetext{
* Correspondence should be addressed to ARRF (alistair.forrest@gmail.com), PC (carninci@ @riken.jp), MR (michael.rehli@ukr.de) or AS (albin@binf.ku.dk).

Author contributions

RA, IH, EA, EV, KL, YC, BL, XZ, MJ, HK, TM, TL, NB, OR, MB, KB, CM, NR, FOB, MR, AS made the computational analysis. JB, MB, TL, HK, NK, JK, HS, MI, CD, ARRF, PC, YH prepared and preprocessed CAGE and/or RNA-seq libraries. EN, PRA, THJ, JB, MB made the knockdown experiments followed by CAGE. CG, CS, LS, JR, DG, ME, MR made the blood cell ChIP experiments, methylation assays and in vitro blood cell validations. TS, CG, YI, YS, EF, SM, YN, ARRF, PC and HS made the HeLa/HepG2 in vitro validations. IME, RA, AS, FM designed and carried out zebrafish in vivo tests. RA, CG, IH, CS, EA, EV, FM, IME, PC, AF, AK, MB, JB, AL, CD, DH, PH, MR, AS interpreted results. RA, CG, IH, EV, IME, JB, FM, DAH, MR, AS wrote the paper with input from all authors.

Competing interests.

The authors declare no competing interests.
} 
Hinxton, European Bioinformatics Institute, Wellcome Trust Genome Campus, Hinxton, Cambridge, CB10 1SD ${ }^{13}$ RIKEN Preventive Medicine and Diagnosis Innovation Program, RIKEN Yokohama Institute, 1-7-22 Suehiro-cho, Tsurumi-ku, Yokohama City, Kanagawa, 230-0045, Japan ${ }^{14}$ Department of Biosciences and Nutrition, Karolinska Institutet, 14183 Huddinge, Stockholm, Sweden. ${ }^{15}$ Department of Clinical Genetics, VU University Medical Center, van der Boechorststraat 7, 1081 BT Amsterdam, Netherlands

\# These authors contributed equally to this work.

\section{SUMMARY}

Enhancers control the correct temporal and cell type-specific activation of gene expression in higher eukaryotes. Knowing their properties, regulatory activity and targets is crucial to understand the regulation of differentiation and homeostasis. We use the FANTOM5 panel of samples covering the majority of human tissues and cell types to produce an atlas of active, in vivo transcribed enhancers. We show that enhancers share properties with CpG-poor mRNA promoters but produce bidirectional, exosome-sensitive, relatively short unspliced RNAs, the generation of which is strongly related to enhancer activity. The atlas is used to compare regulatory programs between different cells at unprecedented depth, identify disease-associated regulatory single nucleotide polymorphisms, and classify cell type-specific and ubiquitous enhancers. We further explore the utility of enhancer redundancy, which explains gene expression strength rather than expression patterns. The online FANTOM5 enhancer atlas represents a unique resource for studies on cell type-specific enhancers and gene regulation.

\section{INTRODUCTION}

Precise regulation of gene expression in time and space is required for development, differentiation and homeostasis in higher organisms ${ }^{1}$. Sequence elements within or near core promoter regions contribute to regulation ${ }^{2}$, but promoter-distal regulatory regions like enhancers are essential in the control of cell type specificity ${ }^{1}$. Enhancers were originally defined as remote elements that increase transcription independent of their orientation, position and distance to a promoter ${ }^{3}$. They were only recently found to initiate RNA polymerase II (RNAPII) transcription, producing so-called eRNAs ${ }^{4}$. Genomic locations of enhancers used by cells can be detected by mapping of chromatin marks and transcription factor binding sites from chromatin immunoprecipitation (ChIP) assays and DNase I hypersensitive sites (DHSs) (reviewed in ref. 1), but there has been no systematic analysis of enhancer usage in the large variety of cell types and tissues present in the human body. Using Cap Analysis of Gene Expression ${ }^{5}$ (CAGE), we show that enhancer activity can be detected through the presence of balanced bidirectional capped transcripts, enabling the identification of enhancers from small primary cell populations. Based upon the FANTOM5 CAGE expression atlas encompassing 432 primary cell, 135 tissue and 241 cell line samples from human ${ }^{6}$, we identify 43,011 enhancer candidates and characterize their activity across the majority of human cell types and tissues. The resulting catalogue of transcribed enhancers enables classification of ubiquitous and cell type-specific enhancers, modeling of 
physical interactions between multiple enhancers and TSSs, and identification of potential disease-associated regulatory single nucleotide polymorphisms (SNPs).

\section{RESULTS}

\section{Bidirectional pairs of capped RNAs identify active enhancers}

The FANTOM5 project has generated a CAGE-based transcription start site (TSS) atlas across a broad panel of primary cells, tissues, and cell lines covering the vast majority of human cell types ${ }^{6}$. Within that dataset, well-studied enhancers often have CAGE peaks delineating nucleosome-deficient regions (NDRs) (Supplementary Fig. 1). To determine whether this is a general enhancer feature, FANTOM5 CAGE (Supplementary Table 1) was superimposed on active (H3K27ac-marked) enhancers defined by HeLa-S3 ENCODE ChIPseq data ${ }^{7}$. CAGE tags showed a bimodal distribution flanking the central P300 peak, with divergent transcription from the enhancer (Fig. 1a). Similar patterns were observed in other cell lines (Supplementary Fig. 2a). Enhancer-associated reverse and forward strand transcription initiation events were, on average, separated by $180 \mathrm{bp}$ and corresponded to nucleosome boundaries (Supplementary Figs 3 and 4). As a class, active HeLa-S3 enhancers had 231-fold more CAGE tags than polycomb-repressed enhancers, suggesting that transcription is a marker for active usage. Indeed, ENCODE-predicted enhancers ${ }^{7}$ with significant reporter activity ${ }^{8}$ had greater CAGE expression levels than those lacking reporter activity $(P<4 \mathrm{e}-22$, Mann-Whitney $\mathrm{U}$ test). A lenient threshold on enhancer expression increased the validation rate of ENCODE enhancers from 27\% to 57\% (Supplementary Fig. 5).

While capped RNAs of protein-coding gene promoters were strongly biased towards the sense direction, similar levels of capped RNA in both directions were detected at enhancers (Fig. 1b, and Supplementary Fig. 2b, c). Thus, bidirectional capped RNAs is a signature feature of active enhancers. On this basis, we identified 43,011 enhancer candidates across 808 human CAGE libraries (see Supplementary Text and Supplementary Figs 6-8). Interestingly, the candidates were depleted of $\mathrm{CpG}$ islands (CGI) and repeats (with the exception of neural stem cells, see ref. 9).

To confirm the activity of newly-identified candidate enhancers, we randomly selected 46 strong, 41 moderate and 36 low activity enhancers (as defined by CAGE tag frequency) and examined their activity using enhancer reporter assays compared to randomly selected untranscribed loci with regulatory potential in HeLa-S3 cells: $15 \mathrm{DHSs}^{10}$, 26 ENCODEpredicted 'strong enhancers'7 and 20 enhancers defined as in Figure 1A (Supplementary Tables 2 and 3). While 67.4-73.9\% of the CAGE-defined enhancers showed significant reporter activity, only $20-33.3 \%$ of the untranscribed candidate regulatory regions were active (Fig. 1c, and Supplementary Fig. 9a). The same trend was observed in HepG2 cells (Supplementary Fig. 10a, b). Corresponding promoter-less constructs showed that the enhancer transcription read-through is negligible (Supplementary Fig. 9b, c). Large fractions of CAGE-defined enhancers overlapped predicted ENCODE 'strong enhancers' or 'TSS' states ( $25 \%$ and $62 \%$, respectively, for HeLa-S3), but there was no substantial difference in validation rates between these classes (Supplementary Fig. 10c, d). In summary, active 
CAGE-defined enhancers were much more likely to be validated in functional assays than untranscribed candidate enhancers defined by histone modifications or DHSs.

\section{Enhancer TSSs share regulatory features with mRNA TSSs but produce short, exosome- sensitive RNAs}

RNA-seq data from matching primary cells and tissues showed that $~ 95 \%$ of RNAs originating from enhancers were unspliced and typically short (median $346 \mathrm{nt}$ ) - a striking difference to mRNAs (19\% unspliced, median 56 nt) (Fig. 2a, and Supplementary Fig. 11ac). Unlike TSSs of mRNAs, which are enriched for predicted 5' splice sites but depleted of downstream polyadenylation (pA) signals ${ }^{11,12}$, enhancers showed no evidence of associated downstream RNA processing motifs, and thus resemble antisense PROMoter uPstream Transcripts (PROMPTs) ${ }^{11}$ (Fig. 2b, and Supplementary Fig. 11d). Most CAGE-defined enhancers gave rise to nuclear (>80\%) and non-polyadenylated ( 90\%) RNAs ${ }^{13}$ (Supplementary Fig. 11e). Based on RNA-seq, few enhancer RNAs overlap exons of known protein-coding genes or lincRNAs (9 and 1 out of 4208 enhancers detected, respectively), suggesting that they are not a substantial source of alternative promoters for known genes (as in ref. 14).

TSS-associated, uncapped small RNAs (TSSa-RNAs), attributed to RNAPII protection and found immediately downstream of mRNA TSSs ${ }^{15,16}$, were detectable in the same positions downstream of enhancer TSSs (Supplementary Fig. 12), indicating that RNAPII initiation at enhancer and mRNA TSSs is similar. Indeed, CAGE-defined enhancer TSSs resembled the proximal position-specific sequence patterns of non-CGI RefSeq TSSs (Fig. 2c, and Supplementary Fig. 13a). Furthermore, de novo motif analysis revealed sequence signatures in CAGE-defined enhancers closely resembling non-CGI promoters (Fig. 2d, and Supplementary Fig. 13b).

Because of the similarity with PROMPTs, we reasoned that capped enhancer RNAs might be rapidly degraded by the exosome. Indeed, siRNA-mediated depletion of the hMTR4 (SKIV2L2) co-factor of the exosome complex resulted in a median 3.14-fold increase of capped enhancer-RNA abundance (Fig. 2e, and Supplementary Fig. 14a, b), but only a negligible increase at mRNA TSSs. This increasing trend is similar to that of PROMPT regions upstream of TSSs, although the increase of enhancer RNAs was significantly higher $(P<4.6 \mathrm{e}-67$, Mann-Whitney U test, Fig. 2e, and Supplementary Fig. 14b, c). Thus, the bidirectional transcriptional activity observed at enhancers is also present at promoters, as suggested previously ${ }^{17}$, but in promoters only the antisense RNA is degraded. Furthermore, the CAGE expression of enhancers in control and hMTR4-depleted cells was proportional (Supplementary Fig. 14d), suggesting that virtually all identified enhancers produce exosome-sensitive RNAs. The number of detectable bidirectional CAGE peaks increased 1.7-fold upon hMTR4 depletion and novel enhancer candidates had on average similar, but weaker, chromatin modification signals compared to control HeLa cells (Supplementary Fig. $14 \mathrm{e})$. 


\section{CAGE expression identifies cell-specific enhancer usage}

To test whether CAGE expression can identify cell type-specific enhancer usage in vivo, ChIP-seq (H3K27ac and H3K4me1), DNA methylation and triplicate CAGE analyses were performed in five primary blood cell types, and compared to published DHS data (www.roadmapepigenomics.org, Supplementary Table 4). CAGE-defined enhancers were strongly supported by proximal H3K4me1/H3K27ac peaks (71\%) and DHSs (87\%) from the same cell type. Conversely, H3K4me1 and H3K27ac supported only 24\% of DHSs distal to promoters and exons and only $4 \%$ of DHSs overlapped CAGE-defined enhancers (Supplementary Fig. 15), suggesting that a minority of promoter-distal DHSs identify enhancers. From the opposite perspective, only 11\% of H3K4me1/H3K27ac loci overlapped CAGE-defined enhancers and untranscribed loci showed weaker ChIP-seq signals than transcribed ones (Supplementary Fig. 16). Moreover, there was a clear correlation between CAGE, DNase I hypersensitivity, H3K4me1 and H3K27ac for CAGE-defined enhancers expressed in blood cells (Fig. 3a). Accordingly, cell type-specific enhancer expression corresponds to cell type-specific histone modifications (Fig. 3b). The majority of selected cell type-specific enhancers could be validated in corresponding cell lines and were associated with cell type-specific DNA demethylation (Supplementary Text, Supplementary Fig. 17, and Supplementary Tables 5-8, see also ref. 18). Thus, bidirectional CAGE pairs are robust predictors for cell type-specific enhancer activity.

\section{An atlas of transcribed enhancers over human cells and tissues}

The FANTOM5 CAGE library collection ${ }^{6}$ enables the dissection of enhancer usage across cell types and tissues comprehensively sampled across the human body. Clustering based upon enhancer expression clearly grouped functionally-related samples together (Fig. 3c, and Supplementary Figs 18 and 19). While fetal and adult tissue often grouped together, two large fetal-specific clusters were identified: one brain-specific (pink) and one with diverse tissues (green). The fetal-brain cluster is associated with enhancers that are located close to known neural developmental genes, including NEUROG2, SCRT2, POU3F2 and MEF2C (Supplementary Fig. 18b), for which gene expression patterns correlate with enhancer RNA abundance across libraries, suggesting regulatory interaction (see below). The results corroborate the functional relevance of these enhancers for tissue-specific gene expression and suggest that they are an important part of the regulatory programs of cellular differentiation and organogenesis.

To confirm that candidate enhancers can drive tissue-specific gene expression in vivo, five evolutionarily conserved CAGE-defined human enhancers (including the POU3F2 and $M E F 2 C$-proximal enhancers identified above) were tested via Tol2-mediated transgenesis in zebrafish embryos. We observed tissue-specific enhancer activity with 3 of 5 fragments, which corresponded to the human enhancer tissue expression (Fig. 4). None of three control fragments without CAGE signal activated the gata2 promoter (Supplementary Table 9). While the sample size is not high enough to reliably estimate the validation rates in zebrafish, the correlation between the enhancer usage profiles in zebrafish to those defined in human by CAGE is notable. 
We grouped the primary cell and tissue samples into larger, mutually exclusive cell type and organ/tissue groups (facets), respectively, with similar function or morphology

(Supplementary Tables 10 and 11). Figure 5 summarizes how many enhancers were detected in each facet and the degree of facet-specific CAGE expression (see also Supplementary Fig. 21). From the data we can draw several conclusions:

First, the majority of detected enhancers within any facet are not restricted to that facet. Exceptions, where facets use a higher fraction of specific enhancers include immune cells, neurons, neural stem cells and hepatocytes amongst the cell type facets, and brain, blood, liver and testis amongst the organ/tissue facets.

Second, despite their apparent promiscuity, enhancers are more generally detected in a much smaller subset of samples than mRNA transcripts (Supplementary Figs 21, and 22a, b), consistent with cell line studies ${ }^{7}$ and the higher specificity of non-coding RNAs (ncRNAs) in general ${ }^{13}$. Facets in which we detect many enhancers typically also have a higher fraction of facet-specific enhancers (Supplementary Fig. 22c, d). Third, the number of detected expressed enhancers and mRNA transcripts is correlated (Supplementary Fig. 21b), but the number of detected expressed gene transcripts (>1TPM) is 19-34 fold larger than the number of detected enhancers with the cutoffs used. Noteworthy exceptions include blood and immune cells, testis, thymus, and spleen, which have high enhancer/gene ratios. Conversely, smooth and skeletal muscle and skin, bone and epithelia-related cells have low ratios.

Differential exosome activity between cell types might affect these results, but there was no correlation between hMTR4 mRNA expression and the number of enhancers detected (Supplementary Fig. 22e, f).

As expected, consensus motifs of known key regulators are over-represented in corresponding facet-specific enhancers, for instance ETS, C/EBP, and NF- $\kappa B$ in monocytespecific enhancers, RFX and SOX in neurons, and HNF1 and HNF4a in hepatocytes (Supplementary Fig. 23). Notably, the AP1 motif appears to be enriched across all facets, perhaps associated with a general role for AP1 in regulating open chromatin ${ }^{19}$.

\section{Expression clustering reveals ubiquitous enhancers}

Hierarchical clustering of enhancers by facet expression revealed a small subset of enhancers ( 241 or 247 , defined by primary cell or tissue facets, respectively) expressed in the large majority of facets (Supplementary Text, Supplementary Figs 24 and 25, and Supplementary Tables 12 and 13). Compared to other enhancers, the ubiquitous (u-) enhancers are 8 times more likely to overlap CGIs and they are twice as conserved (Supplementary Fig. 26a-c). U-enhancers overlap typical chromatin enhancer marks but have higher H3K4me3 signal (Supplementary Fig. 26d). Although they produce significantly longer ncRNAs than other enhancers (median $530 \mathrm{nt}, P<1.5 \mathrm{e}-8$, Mann-Whitney $\mathrm{U}$ test), the transcripts remain predominantly $(\sim 78 \%)$ unspliced and significantly shorter ( $P<4.2 \mathrm{e}-18$, Mann-Whitney U test) than mRNAs (Supplementary Fig. 27-28), do not share exons with known genes, and are exosome-sensitive (Supplementary Fig. 14b). Therefore, it is unlikely that these are novel mRNA promoters. They are also highly enriched for P300 and cohesin ChIP-seq peaks ${ }^{20}$ and RNAPII-mediated ChIA-PET signal ${ }^{21}$ compared to other enhancers (Supplementary Fig. 26d). These results suggest that u-enhancers comprise a 
small but distinct subset of enhancers, which likely has specific regulatory functions utilized by virtually every human cell.

\section{Linking enhancer usage with TSS expression}

A major challenge is to link enhancers to their target genes ${ }^{21,22}$. Uniquely, FANTOM5

CAGE allows for direct comparison between transcriptional activity of the enhancer and of putative target gene TSSs across a diverse set ofhuman cells. Based on pair-wise expression correlation, nearly half (40\%) of the inferred TSS-associated enhancers (Methods) were linked with the nearest TSS, and 64\% of enhancers have at least one correlated TSS within $500 \mathrm{~kb}$. Several $(10,260,15.3 \%)$ associations are supported by ChIA-PET (RNAPIImediated) interaction data ${ }^{21}$, and the supported fraction increases with the correlation threshold (Supplementary Fig. 29a). The fraction of supported associations is 4.8-fold higher than that of associations predicted from DNase hypersensitivity correlations ${ }^{10}(20.6 \%$ vs. $4.3 \%$, at the same correlation threshold), indicating that transcription is a better predictor of regulatory targets than chromatin accessibility. Conserved sequence motifs and ChIP-seq peaks also co-occurred significantly in associated enhancer-promoter pairs (BenjamiHochberg $F D R<0.05$, binomial test), suggesting an additive or synergistic cooperation between enhancers and promoters at RNAPII foci.

On average, a RefSeq TSS was associated with 4.9 enhancers and an enhancer with 2.4 TSSs and we observed different regulatory architectures around genes (Supplementary Fig. 30). For example, at the beta-globin locus the CAGE expression patterns of four locus control region hypersensitive sites are highly correlated (Pearson's $r$ between 0.88 and 0.98 ) with the expression of known target genes ${ }^{23,24} H B G 2, H B D$, and to some extent $H B G 1$.

These observations call for computational models of enhancer regulation, in which multiple enhancers may work in concert to enhance the expression of a gene. To this end, we focused on 2,206 RefSeq TSSs for which the joint expression of nearby enhancers (the closest ten enhancers within $500 \mathrm{~kb}$ ) is highly predictive of the gene expression. Model shrinkage showed that in most cases, only 1-3 enhancers are necessary to explain the expression variance observed in the linked gene, and generally proximal enhancers are more predictive than distal ones (Fig. 6a, Supplementary Fig. 29b-d, and Supplementary Text). One hypothesis explaining the function of multiple enhancers driving the same expression pattern is that they might confer higher transcriptional output of a gene ${ }^{25,26}$. Indeed, the number of highly correlated (redundant) enhancers close to TSSs (Supplementary Methods Online) increased with the observed maximal TSS expression over all libraries (Fig. 6b), implying that these enhancers are redundant in terms of transcription patterns but additive in terms of expression strength. Expression redundancy is also common in genomic clusters of closely spaced enhancers (24\% of 815 identified genomic clusters, Supplementary Table 15). These are associated with TSSs of genes involved in immune and defense responses and, as suggested by a previous study ${ }^{27}$, have a higher expression than other enhancer-associated genes (8-fold increase on average). 


\section{Disease-associated SNPs are enriched in enhancers}

Many disease-associated SNPs are located outside of protein-coding exons and a large proportion of human genes display expression polymorphism ${ }^{28}$. Using the NHGRI GWAS catalog 29 and extending the compilation of lead SNPs with proxy SNPs in strong linkage disequilibrium (similar to refs. 30,31), we identified diseases/traits whose associated SNPs overlapped enhancers, promoters, exons and random regions significantly more than expected by chance (Fisher's Exact Test $P<0.01$, Supplementary Table 16). Diseaseassociated SNPs were over-represented in regulatory regions to a greater extent than in exons (Fig. 6c). For many traits where enriched disease-associated SNPs were within enhancers, enhancer activity was detected in pathologically relevant cell types (Fig. 6d, and Supplementary Figs 31 and 32). Examples include Graves' disease-associated SNPs enriched in enhancers that are expressed predominantly in thyroid tissue, and similarly lymphocytes for chronic lymphocytic leukemia. As a proof of concept, we validated the impact of two disease-associated regulatory SNPs within enhancers (Supplementary Fig. $33)$.

\section{CONCLUSIONS}

The data presented here demonstrate that bidirectional capped RNAs, as measured by CAGE, are robust predictors of enhancer activity in a cell. Transcription is only measured at a fraction of chromatin-defined enhancers and few untranscribed enhancers show potential enhancer activity. This implies that many chromatin-defined enhancers are not regulatory active in that particular cellular state, but may be active in other cells of the same lineage ${ }^{32}$ or are pre-marked for fast regulatory activity upon stimulation ${ }^{33}$. Of course, given the relative instability of enhancer RNAs some chromatin-defined sites may be active but fall below the limits of detection of CAGE.

Our results show that position-specific sequence signals upstream of the transcription initiation sites and the production of small, uncapped, RNAs immediately downstream is present at both enhancers and mRNA promoters, suggesting similar mechanisms of initiation. Previous studies (e.g. refs. 10,34,35) suggested that promoters and enhancers differ in motif composition. This view is not supported by the larger FANTOM5 dataset. Instead, the differences reflect the local GC content since transcribed enhancers tend to harbor GC-poor motifs like non-CGI promoters. Features distinguishing enhancers from mRNA promoters are: i) enhancer RNAs are exosome-sensitive regardless of direction while (sense) mRNAs have a longer half-life than their antisense counterpart; ii) enhancer RNAs are short, unspliced, nuclear and non-polyadenylated and iii) enhancers have downstream pA and 5' splice motif frequencies at genomic background level similar to antisense PROMPTs, while mRNAs are depleted of termination signals and enriched for 5' splice sites ${ }^{11,12}$.

The collection of active enhancers presented here provides a resource that complements the activity of the ENCODE consortium ${ }^{7}$ across a much greater diversity of tissues and cellular. It has clear applications in human genetics, to narrow the search windows for functional association, and for the definition of regulatory networks that underpin the processes of cellular differentiation and organogenesis in human development. 


\section{Data availability}

The FANTOM5 atlas is accessible from http://fantom.gsc.riken.jp/5/top. FANTOM5 CAGE, RNA-seq and sRNA data have been deposited in DDBJ (accession codes DRA000991, DRA001101). Genome browser tracks for enhancers with user-definable expression specificity-constraints can be generated at http://enhancer.binf.ku.dk. Here, pre-defined enhancer tracks and motif finding results are also deposited. Blood cell ChIP-seq data and CAGE data on exosome-depleted HeLa cells have been deposited in the NCBI GEO database (accession codes GSE40668, GSE49834).

\section{Methods (full - for online materials)}

\section{CAGE data}

Single molecule HeliScopeCAGE ${ }^{39}$ data was generated as described elsewhere ${ }^{6}$. We used a set of 432 primary cell, 135 tissue, and 241 cell line samples that passed quality control measures of >500,000 Q20 Delve (Lassmann et al., in prep) mapped CAGE tags, RNA integrity and reproducibility (for further details, see $\operatorname{ref}^{6}$ ).

\section{Proof of concept analysis}

We defined silent and active enhancers from ENCODE HeLa-S3, GM12878 and K562 broad peaks (Broad Institute, Bernstein), downloaded from the UCSC ENCODE repository, according to the co-existence of histone modifications H3K4me1, H3K27ac and $\mathrm{H} 3 \mathrm{~K} 27 \mathrm{me} 3$. Active enhancers were defined as co-localized H3K4me1 and H3K27ac peaks with no H3K27me3 peak, while silent enhancers were considered loci with H3K4me1 and H3K27me3 peaks but no H3K27ac peak. Loci were filtered to be located distant to TSSs (500 bp) and exons (200 bp) of protein-coding genes, multi-exonic non-coding genes and mRNAs (from ENSEMBL, GENCODE (v10), RefSeq and UCSC, downloaded January 12, 2012), and other lncRNAs from a gene-centric set derived from literature ${ }^{40}$ as well as manually annotated sense-antisense pairs (coding-noncoding and noncoding-noncoding sense-antisense pairs) with 5' EST and cDNA support, and 5' ESTs with no locus proteincoding capacity. Transcriptional differences between active and silent enhancer sets were determined by comparing the average number of FANTOM5 CAGE tag 5' ends from the same ENCODE cell lines (pooled triplicates) in a window $+/-300$ bp around the H3K4me1 peak mid points.

The active enhancer sets of HeLa-S3, GM12878 and K562 cells were then centered on proximal (within 200bp) P300 (Stanford, Snyder) ENCODE binding site peaks (joint P300 and GATA1 (Yale) peaks for K562) to derive center positions. FANTOM5 CAGE data from the same ENCODE cell lines (pooled triplicates) were then overlaid these centered enhancer regions and the absence (0) and presence (1) of (one or more) CAGE tag 5' ends in 10bp non-overlapping windows were determined and an average profile was calculated to assess the average bidirectional pattern of transcription at chromatin-derived enhancers.

Pooled CAGE data from all FANTOM5 libraries (described above) were further overlaid with these regions and a directionality score based on the aggregate of CAGE tags falling within $+/-300 \mathrm{bp}$ from the center positions were calculated to determine potential strand 
bias. For comparison, we repeated the same calculations for genomic regions $+/-300 \mathrm{bp}$ around TSSs of RefSeq protein coding genes. Directionality was calculated as $(F-R) /(F+$ $\mathrm{R})$, where $\mathrm{F}$ and $\mathrm{R}$ is the sum of CAGE tags aligned on the forward and reverse strand, respectively. Directionality close to -1 or 1 indicates a unidirectional behavior while 0 indicates perfectly balanced bidirectional transcription.

Positional cross correlations were calculated between reverse and forward CAGE tag 5' ends at ChIP-seq derived active HeLa-S3 and GM12878 enhancer center positions (as determined by P300 peaks) +/-300 bp (max lag 300) to identify their most likely separation. Cross correlations were also calculated in $300 \mathrm{bp}$ windows (max lag 150) flanking the enhancer centers between CAGE 5' ends and ENCODE H2A.Z signals (from the same cell line) for HeLa-S3 and GM12878 as well as between CAGE 5' ends and ENCODE GM12878 nucleosome MNase-seq 5' ends (9 pooled replicates). In the latter analysis, correlations were made using reads on the same strand. Pooled, unique CAGE tags (in which only one CAGE tag per bp was counted) were considered in all correlation analyses and enhancers were weighed according to the aggregated signal before subsequent averaging over lags not to make any library or enhancer have an undue influence.

\section{Reporter activity of ENCODE enhancers in relation to transcriptional status}

We used published ${ }^{8}$ results on a massively parallel reporter assay measuring the activity of ENCODE-predicted enhancers in HepG2 and K562 cells. All results on non-scrambled sequences were considered, regardless of the level of conservation. 198 out of 738 tested K562 enhancers and 307 out of 1136 tested HepG2 enhancers had significant enhancer reporter activity (as determined by the original publication). We determined the expression in 401bp windows centered on mid points of ENCODE-predicted enhancers using FANTOM5 CAGE from the same cell lines. We further calculated the false discovery rate after a minimum expression threshold in the interval $[0,0.5] \mathrm{TPM}$, as the fraction of nonsignificant enhancers among those fulfilling the expression cutoff.

\section{Identification of bidirectionally transcribed loci}

Bidirectionally transcribed loci were defined from a set of 1,714,047 forward and 1,597,186 reverse strand CAGE tag clusters (TCs) supported by at least two CAGE tags in at least one sample (TCs defined in ${ }^{6}$ ). Only TCs not overlapping antisense TCs were used. We identified 1,261,036 divergent (reverse-forward) TC pairs separated by at most $400 \mathrm{bp}$ and merged all such pairs containing the same TC, while at the same time avoiding overlapping forward and reverse strand transcribed regions (prioritization by expression ranking), which resulted in 200,171 bidirectional loci (procedure illustrated in Supplementary Figure 6a). A center position was defined for each bidirectional locus as the mid position between the rightmost reverse strand TC and leftmost forward strand TC included in the merged bidirectional pair. Each bidirectional locus was further associated with two $200 \mathrm{bp}$ regions immediately flanking the center position, one (left) for reverse strand transcription and one (right) for forward strand transcription, in a divergent manner. The merged bidirectional pairs were further required to be bidirectionally transcribed (CAGE tags supporting both windows flanking the center) in at least one individual sample, and to have a greater aggregate of reverse CAGE tags (over all FANTOM5 samples) than forward CAGE tags in 
the $200 \mathrm{bp}$ region associated with reverse strand transcription, and vice versa. These filtering steps resulted in 78,555 bidirectionally transcribed loci.

\section{Expression quantification of bidirectionally transcribed loci and prediction of enhancers}

We quantified the expression of bidirectional loci for each strand and $200 \mathrm{bp}$ flanking window in each of the 432 primary cell, 135 tissue and 241 cell line samples separately by counting the CAGE tags whose 5' ends were located within these windows. The expression values of both flanking windows were normalized by converting tag counts to tags per million mapped reads (TPM) and further normalization between samples was done using the RLE normalization procedure in edge $\mathrm{R}^{41}$. The number of CAGE tags aligned on ChrM was subtracted from the total number of aligned CAGE tags in each library before normalization. The normalized expression values from both windows were used to calculate a sample-set wide directionality score, $D$, for each enhancer over aggregated normalized reverse, $R$, and forward, $F$, strand expression values across all samples (Supplementary Fig. 6a); $D=(F$ $-R) /(F+R)$. $D$ ranges between -1 and 1 and specifies the bias in expression to reverse and forward strand, respectively ( $D=0$ means $50 \%$ reverse and 50\% forward strand expression, while $\operatorname{abs}(D)$ close to 1 indicates unidirectional transcription). A directionality score calculated from pooled data is a good estimate of sample directionality (Supplementary Fig. 6b). Each bidirectional locus was assigned one expression value for each sample by summing the normalized expression of the two flanking windows.

Bidirectional loci were further filtered to have low, non-promoter-like, directionality scores $(\operatorname{abs}(D)<0.8)$ and to be located distant to TSSs and exons of protein- and non-coding genes (see 'Proof of concept analysis' above for details). This resulted in a final set of 43,011 putative enhancers.

We further tested whether the expression level for each sample and candidate enhancer was significantly greater than the genomic background (see construction of random genomic background regions below). A $P$-value was calculated for each enhancer expression value for each primary cell, tissue and cell line sample by counting the fraction of random genomic regions with greater expression level in the same sample. Enhancers with $P$-values less than 0.001 and Benjamini-Hochberg adjusted $F D R<0.05$ was considered transcribed in that sample. This analysis yielded binary expression values, which were used for constructing enhancer sets associated with each sample. In total, 38,554 enhancers were transcribed at a significant expression level in at least one primary cell or tissue sample. Below, we refer to this set as the 'robust set' of enhancers and indicate whenever it was used. For all analyses, we use the whole ('permissive') set of 43,011 enhancers if not otherwise mentioned.

\section{Construction of random genomic background regions}

We randomly sampled 100,000 genomic regions of $401 \mathrm{bp}$ that were distal to TSSs and exons of known genes (same as the filtering procedure described above for bidirectionally transcribed loci). These were further filtered to not overlap with our set of 43,011 predicted enhancers, which yielded 98,942 random genomic regions whose expression levels were quantified and normalized in the same manner as described for bidirectional loci (above). 


\section{Correlation between ENCODE epigenomic data and CAGE-defined enhancers}

Using the UCSC ENCODE repository data (downloaded and pooled March 26 2012), we assessed the signal of RNA Polymerase II (RNAPII), the pooled transcription factor super track (all TFs), CCCTC-binding factor (CTCF), E1A binding protein P300, DNase I hypersensitive sites (DHSs) and two histone marks: H3K4me1 and H3K27ac around enhancers, TSSs and random genomic sites.

\section{Large scale enhancer reporter validations}

We randomly selected 125 CAGE-defined enhancers with significantly higher expression than random genomic regions in at least two out of three HeLa-S3 replicates. These were grouped according to Hela-S3 expression tertiles: (low (36), mid-level (41) and strong (46). These could be split up further according to overlap (mid position) with combined ENCODE (release Jan 2011) segmentations of Segway ${ }^{42}$ and ChromHMM ${ }^{43}$ chromatin state prediction: 25, 27, and 14 strongly, mid-level and lowly expressed CAGE enhancer overlapped ENCODE state 'E' ('strong enhancer') while 21, 16, and 22 strongly, mid-level and lowly expressed CAGE enhancer overlapped ENCODE state 'TSS'.

We further randomly selected 26 and 15 untranscribed (negligible amount of overlapping FANTOM5 HeLa-S3 CAGE tags) 500bp regions centered on mid positions of HeLa-S3 E states and HeLa-S3 ENCODE DHSs. Two literature-derived ${ }^{44} \mathrm{HeLa}-\mathrm{S} 3$ positive enhancers and 4 random regions (see 'Construction of random genomic background regions') were used for comparison. For comparison, we also randomly selected 20 manually defined untranscribed HeLa-S3 chromatin-defined active enhancers (see 'Proof of concept analysis').

PCR primers for the amplification of enhancer and control regions were designed using the PerlPrimer tool ${ }^{45}$, and purchased from Operon Ltd. Primers included BamHI or SaII restriction sites for cloning and sequences are listed in Supplementary Tables 2 and 3. Control fragments ranged between 420-1452bp. Enhancer fragments usually included a $500 \mathrm{bp}$ window around the mid point of our predicted enhancers and depending on the availability of unique primer sequences, enhancer fragments ranged between 470-840bp.

We inserted an EF1a basal promoter fragment into HindIII and NheI sites of the multiple cloning site in pGL4.10 (Promega) to construct a basal pGL4.10EF1a backbone. We next removed the the BamHI and SaIl containing fragment located at downstream of the SV40 late poly(A) signal of the original pGL4.10 vector backbone, and re-inserted the fragment at the SpeI site that is located upstream of the synthetic poly(A) signal/transcriptional pause site to generate modified versions of pGL4.10EF1a and pGL4.10 (see Supplementary Figure 9d).

Enhancer and control regions were PCR-amplified using KOD plus polymerase (TOYOBO) from HEK-293T gDNA, digested with BamHI and Sall (TAKARA BIO), and purified using the E-Gel ${ }^{\circledR}$ SizeSelect ${ }^{\mathrm{TM}}$ system (Life Technologies). Five $\mu \mathrm{l}$ of purified PCR products were ligated with $100 \mathrm{ng}$ of the BamHI- and SaII-digested modified pGL4.10EF1a and pGL4.10 plasmids using Ligation-high (TOYOBO), and transformed into DH5a competent cells 
(TOYOBO). Correct insertion of the PCR products into the plasmids was checked by colony PCR. Vectors were purified using the QIAGEN Plasmid Plus 96 Miniprep Kit (QIAGEN).

HeLa-S3 cells (JCRB Cell Bank) were cultured in MEM (WAKO) supplemented with 10\% FBS (NICHIREI BIOSCIENCE INC., Lot No. 7G0031), 100 Units/mL penicillin and 100 $\mu \mathrm{g} / \mathrm{mL}$ streptomycin (both Life Technologies). HepG2 Cells (RIKEN BRC) were cultured in DMEM (Life Technologies) supplemented with 10\% FBS (NICHIREI BIOSCIENCE INC., Lot No. 7G0031), and MEM (WAKO) supplemented with 10\% FBS (NICHIREI BIOSCIENCE INC., Lot No. 7G0031), 100 Units penicillin and $100 \mu \mathrm{g} / \mathrm{mL}$ streptomycin (Life Technologies). Cell lines were seeded into 96 well plates at a density of $7.5 \times 10^{3}$ cells/ well one day before transfection. Firefly luciferase reporter plasmids (190 ng) and $10 \mathrm{ng}$ of pGL4.73 renilla luciferase plasmid (Promega) were co-transfected into HepG2 or HeLa-S3 cells using Lipofectamine (Life Technologies) according to the manufacturer's instruction. Each transfection was independently performed three times. After 24 hours, the luciferase activities were measured by GloMax 96 Microplate luminometer (Promega) using the Dualglo luciferase assay system (Promega) according to the manufacturer's instruction.

\section{Sequence motif analysis on global CAGE enhancer and promoter sets}

To compare motif signatures characterizing bidirectionally transcribed enhancers (permissive set) with those of CAGE-defined promoters, we used the set of 184,827 robust human CAGE clusters defined by ${ }^{6}$ separated into 61,322 CGI and 123,505 nonCGIassociated clusters. We made further subsets of these CAGE clusters, contingent on their overlap with annotated TSSs from Refseq and Gencode. We merged overlapping extended CAGE clusters $(-300,+50$; based on the robust cluster set; average size nonCGI: $422 \mathrm{bp}$; average size CGI: $544 \mathrm{bp}$ ) contingent on CGI status and subtracted CAGE cluster regions that overlapped with extended enhancers (mid position $+/-200 \mathrm{bp}$ ).

This created five sets of regions representing non-overlapping bidirectional enhancers, nonCGI promoters and CGI promoters (annotated and full sets for the two latter ones). Motif enrichment was analyzed using $\mathrm{HOMER}^{36}$ version 3 , a suite of tools for motif discovery and next-generation sequencing analysis (http://biowhat.ucsd.edu/homer/). Sequences of the three region sets (enhancers, nonCGI and CGI promoters) were compared to equal numbers of randomly selected genomic fragments of the average region size, matched for GC content and autonormalized to remove bias from lower-order oligo sequences. After masking repeats, motif enrichment was calculated using the cumulative binomial distribution by considering the total number of target and background sequence regions containing at least one instance of the motif. One hundred motifs were searched for a range of motif lengths (7-14 bp) resulting in a set of 800 de novo motifs per set. After filtering redundant motifs, the top 50 motifs resulting from each search were combined, remapped and ranked according to enrichment (depletion) in the enhancer set. In parallel, we also used HOMER to calculate the enrichment of ChIP-seq derived known transcription factor motifs. Motif collections including search parameters are deposited in a web database at http:// enhancer.binf.ku.dk. Histograms of PhastCons scores were generated using the annotation tool in HOMER. 


\section{Analysis of splice site and termination signals downstream of CAGE enhancer TSSs and promoter TSSs}

To identify motifs downstream of TSSs potentially differing between the structurally related bidirectionally transcribed enhancer TSSs and nonCGI-associated promoter TSSs, we extracted 600bp regions downstream of each TSS and performed comparative de novo motif searches using HOMER. Here, we analyzed one set using the other set as background (corrected for region size, matched for GC content and autonormalized) to calculate motif enrichment only on the given strand. The top motif enriched downstream of nCGI promoters was the 5'-splice site motif. Genomic distributions of the enriched splice site motif, as well as the AATAAA termination signal were generated using HOMER.

\section{RNA-seq samples and library preparation}

Prior to preparation of sequencing libraries, rRNA was removed by poly(A)+ selection (CD19+ B-cells, CD8+ T-cells, 500 ng) or rRNA depletion (fetal heart, 1 ug). Poly(A) ${ }^{+}$ selection was done twice by using Dynabeads Oligo(dT) 25 (Life Technologies) according to the manufacturer's manual. rRNA depletion was done by using Ribo-Zero rRNA removal kit (Epicentre, Illumina) according to the manual. The treated RNA was dissolved in $20 \mu \mathrm{L}$ water.

The pretreated RNA was then fragmented by heating at $70^{\circ} \mathrm{C}$ for $3.5 \mathrm{~min}$ in fragmentation buffer (Ambion), followed by immediate chilling on ice and addition of $1 \mu \mathrm{l}$ of Stop solution. Fragmented RNA was purified with the RNeasy MinElute kit (Qiagen) following the instructions of the manufacturer except $675 \mu \mathrm{L}$ of $100 \%$ ethanol is used in step two, instead of $500 \mu$ l. Purified RNA was dephosphorylated in phosphatase buffer (New England Biolabs) with $5 \mathrm{U}$ of Antarctic phosphatase (New England Biolabs) and $40 \mathrm{U}$ of RNaseOut (Life Technologies) at $37^{\circ} \mathrm{C}$ for $30 \mathrm{~min}$ followed by $5 \mathrm{~min}$ at $65^{\circ} \mathrm{C}$. After chilling on ice RNA was phosphorylated by addition of the following reagents; $5 \mu$ of $10 \times$ PNK buffer, 20 $\mathrm{U}$ of T4 polynucleotide kinase (New England Biolabs), $5 \mu \mathrm{l}$ of $10 \mathrm{mM}$ ATP (Epicentre, Illumina), $40 \mathrm{U}$ of RNaseOut, $17 \mu \mathrm{l}$ of water. The reaction was incubated at $37^{\circ} \mathrm{C}$ for $60 \mathrm{~min}$. Phosphorylated RNA was purified with the RNeasy MinElute kit (Qiagen) as described above. Purified RNA was concentrated to $6 \mu$ by vacuum centrifugation on a SpeedVac (Eppendorf). One $\mu \mathrm{l}$ of $2 \mu \mathrm{M}$ pre-adenylated 3' DNA adaptor, 5'-App/ATC TCG TAT GCC GTC TTC TGC TTG-3' was added to the concentrated RNA. After incubation at $70^{\circ} \mathrm{C}$ for 2 min followed by chilling on ice for $2 \mathrm{~min}$, the following reagents were added to ligate the adapter at the 3' end of the RNA; $1 \mu$ of 10× T4 RNA ligase 2 truncated buffer, $0.8 \mu$ of $100 \mathrm{mM} \mathrm{MgCl} 2,20 \mathrm{U}$ of RNaseOUT and $200 \mathrm{U}$ of RNA ligase 2 truncated (New England Biolabs). After the incubation at $20^{\circ} \mathrm{C}$ for $60 \mathrm{~min}, 1 \mu \mathrm{l}$ of heat-denatured $5 \mu \mathrm{M} 5^{\prime}$ RNA adapter, 5'-guu cag agu ucu aca guc cga cga ucg aaa-3' was ligated with 3' adapter ligation products with $20 \mathrm{U}$ of T4 RNA ligase 1 (New England Biolabs) and $1 \mu \mathrm{l}$ of $10 \mathrm{mM}$ ATP (New England Biolabs) at $20^{\circ} \mathrm{C}$ for 60 min. $4 \mu \mathrm{l}$ of adapter ligated RNA was mixed with $1 \mu \mathrm{l}$ of $20 \mu \mathrm{M}$ RT Primer, 5'-CAA GCA GAA GAC GGC ATA CGA-3', followed by incubation at $70^{\circ} \mathrm{C}$ for $2 \mathrm{~min}$, and immediately kept on ice. RT reaction was done with $2 \mu \mathrm{l} 5 \times$ Prime Script buffer, $1 \mu \mathrm{l}$ of $10 \mathrm{mM}$ dNTP, $20 \mathrm{U}$ of RNaseOUT and $200 \mathrm{U}$ of PrimeScript Reverse Transcriptase (TakaraBIO) at $44^{\circ} \mathrm{C}$ for $30 \mathrm{~min}$. The cDNA product was amplified by PCR with $10 \mu \mathrm{l}$ of $5 \times$ HF buffer, $1.25 \mu \mathrm{l}$ of $10 \mathrm{mM}$ each dNTP mix, $2 \mu \mathrm{l}$ of $10 \mu \mathrm{M}$ FWD primer, 
5'-AAT GAT ACG GCG ACC ACC GAC AGG TTC AGA GTT CTA CAG TCC GA-3', 2 $\mu \mathrm{l}$ of RT primer and $1 \mathrm{U}$ of Phusion High-Fidelity DNA Polymerase (New England Biolabs). PCR was carried out in a total volume of $50 \mu \mathrm{l}$ with the following thermal program; $98^{\circ} \mathrm{C}$ for $30 \mathrm{sec}, 12 \mathrm{PCR}$ cycles of $10 \mathrm{sec}$ at $98^{\circ} \mathrm{C}, 30 \mathrm{sec}$ at $60^{\circ} \mathrm{C}$, and $15 \mathrm{sec}$ at $72^{\circ} \mathrm{C}$, followed by at $72^{\circ} \mathrm{C}$ for $5 \mathrm{~min}$ and then kept at $4^{\circ} \mathrm{C}$. Remaining PCR primers were removed twice by using 1.2 volumes of AMPure XP beads (Beckman Coulter). The resulting libraries were checked for size and concentration by BioAnalyzer (Agilent) using the High-Sensitivity DNA Kit (Agilent). Qualified sequencing libraries were loaded on the HiSeq2000 (Illumina) using the custom sequencing primer, 5'-CGA CAG GTT CAG AGT TCT ACA GTC CGA CGA TCG AAA-3'.

All RNA-seq samples profiled in this study were also profiled in the FANTOM5 promoterome manuscript and are described in detail there ${ }^{6}$. Briefly all human samples used in the project were either exempted material (available in public collections or commercially available), or provided under informed consent. All non-exempt material is covered under RIKEN Yokohama Ethics applications (H17-34 and H21-14). For the samples profiled by RNA-seq, the human fetal heart RNA was purchased from Clontech (Cat no.636583). CD19+ B-cells and CD8+ T-cells were isolated using the pluriBead ${ }^{\circledR}$ system (huCD4/CD8 cascade and huCD19 single; PluriSelect). RNA was then extracted using the miRNeasy kit (Qiagen).

\section{RNA-seq mapping and transcript assembly}

Single-end 100bp long reads from libraries originating from the similar cell sources (all six "CD19+ B cells" libraries, all six "CD8+ T cells" libraries and one "Fetal heart" library) were processed together via the Moirai pipeline (Hasegawa et al., manuscript in preparation). The processing steps implemented within the Moirai pipeline included 1) raw sequenced reads PolyA tail and "CTGTAGGCACCATCAAT" adaptor clipping using FASTQ/A Clipper from FASTX-Toolkit (http://hannonlab.cshl.edu/fastx_toolkit/), 2) removal of sequenced reads containing "N" and sequences similar to ribosomal RNA using rRNAdust version 1.02 (Lassmann et al., manuscript in preparation), and 3) mapping the resulting reads against the $\mathrm{hg} 19$ human genome using TopHat ${ }^{46}$ (version 1.4.1) using both TopHat de novo junction finding mode and known exon-exon junctions extracted from GENCODE V10, with all the other parameters set to their default values. Mapped reads flagged as PCR duplicates were removed and the remaining TopHat aligned reads were then assembled using Cufflinks ${ }^{47}$ (version 1.3.0) with Cufflinks parameters set to their default values.

\section{Assessment of lengths of RNAs emanating from enhancers and promoters}

All Cufflinks assembled transcripts, whose 5'ends, regardless of strand, were located within the outer boundaries of CAGE enhancers or, on the same strand, within 200bp (upstream or downstream) of a GENCODE (v10) protein-coding TSS were considered for further analysis. For these Cufflinks transcripts we calculated their (intron-less) RNA length, (possibly intron-containing) genomic length as the genomic distance between their 5' and 3' ends, as well as their number of exons. Exons of Cufflinks transcripts with 5' ends in enhancers were further checked for at least 50\% (reciprocal) overlap with exons of 
GENCODE (v10) known, level 1, protein-coding genes and lincRNAs. We repeated the same analysis specifically for u-enhancers.

\section{Small RNA library preparation and mapping}

Short RNA-seq sequencing libraries were prepared as 24-plex using the TruSeq Small RNA Sample Prep Kit (Illumina) following the manufacturer's manual. All starting sources were 1 $\mu \mathrm{g}$ of total RNA. The prepared sequencing libraries were loaded on a HiSeq2000 (Illumina). All samples profiled in this study were also profiled in the FANTOM5 promoterome paper 6 and are described in detail there. Briefly, all human samples used in the project were either exempted material (available in public collections or commercially available), or provided under informed consent. All non-exempt material is covered under RIKEN Yokohama Ethics applications (H17-34 and H21-14). For the samples profiled by sRNA-seq, the human fetal heart RNA was purchased from Clontech (Cat no.636583). CD19+ B-cells and CD8+ T-cells were isolated using the pluriBead ${ }^{\circledR}$ system (huCD4/CD8 cascade and huCD19 single; PluriSelect). RNA was then extracted using the miRNeasy kit (Qiagen).

Short RNAs were profiled using the Truseq protocol from Illumina, using an 8-plex. The 8plex was first split by barcode and the resulting FASTQ sequences trimmed of the 3' adapter sequence. Sequences with low quality base N were removed. Ribsomal RNA sequences were then removed using the rRNAdust program. Remaining reads were then mapped using BWA version is $0.5 .9(\mathrm{r} 16)$ and multimappers were randomly assigned.

\section{Analysis of small RNAs at enhancer TSSs and promoter TSSs}

5' and 3' ends of mapped sRNAs as well as pooled CAGE 5' ends were overlaid windows of $601 \mathrm{bp}$ centered on forward strand summits of enhancer-defining CAGE tag clusters and sense strand summits in promoters of RefSeq protein-coding genes. The average crosscorrelation between CAGE 5' ends and sRNA 3' ends were calculated in these windows allowing a max lag of 300 . For footprint plots, reads mapping to the same genomic locations were only counted once not to make any library or genomic region have an undue influence.

\section{HeLa cells culturing and hMTR4 depletion}

HeLa cells were grown in DMEM medium supplemented with $10 \%$ fetal bovine serum at $37^{\circ} \mathrm{C}$ and $5 \% \mathrm{CO}_{2}$. siRNA-mediated knockdown of either EGFP(control), and hMTR4 (SKIV2L2) were performed using $22 \mathrm{nM}$ of siRNA and Lipofectamin2000 (Invitrogen) as transfecting agent. A second hit of $22 \mathrm{nM}$ siRNA was given after $48 \mathrm{~h}$. Cells were harvested an additional $48 \mathrm{~h}$ after the second hit, and protein depletion was verified by western blotting analysis as described elsewhere ${ }^{48}$. The following siRNA sequences were used:

egfp GACGUAAACGGCCACAAGU[dT][dT]

egfp_as ACUUGUGGCCGUUUACGUC[dT][dT]

hMTR4 CAAUUAAGGCUCUGAGUAA[dT][dT]

hMTR4_as UUACUCAGAGCCUUAAUUG[dT][dT] 


\section{HeLa CAGE library preparations and data processing}

CAGE libraries were prepared from $5 \mu$ o of total RNA purified from $2 \times 10^{6} \mathrm{HeLa}$ cells using the Purelink mini kit (Ambion) with 1\% 2-Mercaptoethanol (Sigma) and on-column DNAse I treatment (Ambion) as recommended by manufacturer. CAGE libraries were prepared according as described previously ${ }^{49}$. Prior to sequencing four libraries with different barcodes were pooled and applied to the same sequencing lane. The libraries were sequenced on a HiSeq2000 instrument (Illumina). To compensate for the low complexity in 5 'end of the CAGE libraries 30\% Phi-X spike-in were added to each sequencing lane as recommended by Illumina. CAGE reads were assigned to their respective originating sample according to identically matching barcodes. Assigned reads were trimmed to remove linker sequences and subsequently filtered for a minimum sequencing quality of 30 in $50 \%$ of the bases using the FASTX-Toolkit (http://hannonlab.cshl.edu/fastx_toolkit/). Mapping to the human genome (hg19) was performed using Bowtie ${ }^{50}$ (version 0.12.7), allowing for multiple good alignments and subsequently filtering for uniquely mapping reads. Reads that mapped to unplaced chromosome patches or chrM were discarded.

\section{Assessment of degradation rates of RNAs emanating from CAGE enhancers and promoters}

Bidirectionally transcribed loci were identified in the same way as with pooled FANTOM5 CAGE libraries (see 'Identification of bidirectionally transcribed loci' and 'Expression quantification of bidirectionally transcribed loci and prediction of enhancers' above) from tag clusters (as defined in ${ }^{51}$ ) derived from pooled HeLa CAGE (mock treated control, hMTR4) libraries. From 5,892 bidirectional loci distant to TSSs and exons, 4196 were predicted to be enhancers based on balanced directionality of transcription of which 3,896 had significantly greater expression than random genomic regions in at least one library. These were then overlapped with the whole set of FANTOM5 CAGE enhancers to estimate the fraction of unseen transcribed enhancers.

The expression fold change of HeLa depleted of hMTR4 compared to mock treated control were assessed and compared between expressed HeLa CAGE enhancers, promoters of RefSeq protein-coding genes in general and broken up into $\mathrm{CpG}$ and non-CpG promoters, and ubiquitous FANTOM5 CAGE enhancers.

We further calculated the average footprints of $\mathrm{H} 3 \mathrm{~K} 4 \mathrm{me} 1, \mathrm{H} 3 \mathrm{~K} 4 \mathrm{me} 2$ and $\mathrm{H} 3 \mathrm{~K} 27 \mathrm{ac}$ from ENCODE (Broad, Bernstein) signal files in $601 \mathrm{bp}$ windows centered on mid points of enhancers identified in HeLa cells and those that were novel in hMTR4-.

\section{Purification of blood cell types}

Peripheral blood mononuclear cells were isolated from leukapheresis products of healthy volunteers by density gradient centrifugation over Ficoll/Hypaque (Biochrom AG, Germany). Collection of blood cells from healthy donors was performed in compliance with the Helsinki Declaration. All donors signed an informed consent. The leukapheresis procedure and subsequent purification of peripheral blood cells was approved by the local ethical committee (reference number 92-1782 and 09/066c). CD4+ cells were enriched using magnetically labeled human CD4 MicroBeads (Miltenyi Biotec,Germany) and the Midi- 
MACS system (Miltenyi Biotec). The CD4+ fraction was stained with CD4 FITC (Becton Dickinson, cat no. 345768), CD25 PE (Becton Dickinson, cat no. 341011) and CD45RA APC and CD3+CD4+CD25- T cells were sorted on a FACS-Aria high-speed cell sorter (BD Biosciences, Germany). CD8+ cells were enriched using magnetically labeled human CD8 MicroBeads (Miltenyi). The CD8+ fraction was stained with CD3 FITC (Becton Dickinson, cat no 345763) and CD8 APC (Becton Dickinson, cat no. 345775) and sorted for CD3+CD8+ T cells. CD19+ and CD56+ cells were enriched from the CD8- fraction using magnetically labeled human CD19 and CD56 MicroBeads (Miltenyi). Enriched cells were stained with CD3 FITC (Becton Dickinson,cat no. 345763), CD19 PE (Becton Dickinson, cat no. 345777) and CD56 APC (Becton Dickinson, cat no.341027) and sorted into CD3+CD19+ B cells and CD3+CD56+ NK cells. Purification of blood monocytes is described elsewhere ${ }^{52}$.

\section{Generation of ChIP data for blood cells}

Chromatin was obtained from CD4+CD25- T cells, CD8+ T cells, CD19+ B cells, and CD56+ NK cells of two healthy male donors each. Chromatin immunoprecipitation (ChIP) for $\mathrm{H} 3 \mathrm{~K} 4 \mathrm{me} 1$ and $\mathrm{H} 3 \mathrm{~K} 27 \mathrm{ac}$ and library construction were done essentially as described elsewhere ${ }^{52}$. Sequence tags were mapped to the current human reference sequence (GRCh37/hg19) using Bowtie ${ }^{50}$ and only uniquely mapped tags were used for downstream analyses. H3K4me1 and H3K27ac ChIP-seq data for CD14+ monocytes was generated elsewhere ${ }^{52}$. Complementary DNase hypersensitivity sequencing data was obtained from the Epigenetics Roadmap project (www.roadmapepigenomics.org) and mapped as above. Blood cell ChIP-seq data have been deposited with the NCBI GEO database (accession code GSE40668) and UCSC Genome Browser track hub data of the entire blood cell data set can be found at http://www.ag-rehli.de/NGSdata.htm. Also see Supplementary Table 4.

\section{Clustering of blood cell CAGE and epigenetics data}

CAGE samples corresponding to CD4+, CD8+, B cells, NK cells and monocytes were selected in triplicates from among the set of primary cell samples. Based on the total set of 43,011 permissive enhancers, a subset of 6,609 blood-expressed enhancers was defined as being significantly expressed above genomic background (described above) in at least two of the triplicate samples for at least one blood cell type. This subset of enhancers was clustered for heat map visualization using complete linkage agglomerative hierarchical clustering based on enhancer usage per cell type (binary matrix) and Manhattan distance.

Enhancers were defined as being specifically expressed in one blood cell type if having a pairwise $\log 2$ fold change $>1.5$ with respect to the other four blood cell types. The fold change was calculated based on the mean expression over triplicate samples per cell type. Footprints for DNase I hypersensitivity (DHS), H3K4me1 and H3K27ac were calculated per cell type-specific enhancer set and cell type by extension of reads to $200 \mathrm{bp}$ and overlap aggregation for a window of $+/-1 \mathrm{~kb}$ around enhancer midpoint as the mean TPM signal over all enhancers in that specific subset.

Peak-calling was done using MACS2 ${ }^{53}$ on pooled data for DHS, H3K4me1 and H3K27ac. Per cell type, peaks were regarded as significant if the peak summit fell within the upper 1 
percentile of the background signal (max values in 92,604 random $1 \mathrm{~kb}$ non-TSS nonenhancer regions). DHS regions were defined as $+/-500$ bp around peak summits. Since ChIP-Seq signals for H3K4me1 and H3K27ac often form bimodal peaks around enhancer sites, peak regions were defined as merged regions resulting from overlapping $+/-500 \mathrm{bp}$ regions around MACS2 called peak summits.

\section{Transient enhancer-reporter assays in blood cells}

Selected blood cell type-specific enhancer regions (ranging from 800-1200 bp) were PCRamplified from human genomic DNA and cloned directly into the CpG-free pCpGLCMV/EF1 vector ${ }^{54,55}$ replacing the CMV enhancer with the DMR regions. Primer sequences are given in Supplementary Table 5. All inserts were verified by sequencing. For transient transfections, plasmids were isolated and purified using the EndoFree Plasmid Kit (Qiagen). Each luciferase construct was transiently transfected into three model cell lines (the monocytic THP-1 cell line, the Jurkat T cell line, and the B cell lymphoma cell line DAUDI). THP-1 and DAUDI cells were transfected using DEAE-dextran with $200 \mathrm{ng}$ reporter plasmid and $10 \mathrm{ng}$ Renilla control vector essentially as described ${ }^{56}$. Jurkat cells were transfected as described elsewhere ${ }^{54}$. The transfected cell lines were cultivated for 48 $\mathrm{h}$, harvested, and cell lysates were assayed for firefly and Renilla luciferase activity using the Dual Luciferase Reporter Assay System (Promega) on a Lumat LB9501 (Berthold, Wildbach, Germany). Firefly luciferase activity of individual transfections was normalized against Renilla luciferase activity. Transfections correspond to at least three independent experiments measured in duplicates.

To correct enhancer activity for the amount of read-through that is potentially generated from the enhancer TSS, we additionally generated constructs lacking the basal EF1a promoter for all B cell-specific constructs. Relative luciferase activities generated by readthrough activity were subtracted from the activity of enhancer/EF1 constructs to reveal 'true' enhancer activities of individual regions. To further determine the position and activity of reporter TSS, 5' RACE-PCR for the luciferase gene was performed as follows: RNA of transfected DAUDI cells was reverse transcribed using the SMARTer ${ }^{\mathrm{TM}}$ RACE cDNA Amplification Kit (Clontech, France) according to the manufacturers' instructions. Rapid Amplification of luciferase 5' cDNA ends (5' RACE) was performed with the Advantage 2 Polymerase System (Clontech) and a LUC specific primer (5'-CAT GGC TTC TGC CAG CCT CAC AGA CAT C-3') using the recommended touchdown-PCR program. $15 \mu$ l of the PCR products were analyzed by agarose gel electrophoresis (2.5\%). In addition, fragments were cloned using the StrataClone PCR cloning Kit (Agilent) according to the manufacturers' instructions and sequenced (Life Technologies, Germany).

\section{Mass spectrometry analysis of bisulfite-converted DNA}

For the set of genomic regions that were also used in transient enhancer-reporter assays, PCR primers were designed using the MethPrimer web tool ${ }^{57}$ and purchased from SigmaAldrich (Munich, Germany) (for sequences see Supplementary Table 7). Sodium bisulfite conversion was performed using the EZ DNA methylation kit (Zymo Research, California, USA) using 200-1000 ng of genomic DNA from CD4+CD25- T cells, CD8+ T cells, CD14+ monocytes, CD19+ B cells, and CD56+ NK cells (two donors each) and an 
alternative conversion protocol. Amplification of target regions was followed by SAP treatment, reverse transcription and subsequent RNA base-specific cleavage (MassCLEAVE, San Diego, CA) as previously described ${ }^{58}$. Cleavage products were loaded onto silicon chips (SpectroCHIP, CA) and analyzed by MALDI-TOF mass spectrometry (MassARRAY Compact MALDI-TOF, U.S.Sequenom, San Diego, CA). Methylation was quantified from mass spectra using the Epityper software (Sequenom, U.S), and averaging methylation levels of $\mathrm{CpG}$ dinucleotides located in the central DNase hypersensitive (nucleosome-free) region that is flanked by CAGE clusters. The methylation data for individual CpGs are provided in Supplementary Table 8 .

\section{Definition of expression facets and differentially expressed 'specific' facets}

Cell and UBERON ontology term mappings were extracted from the FANTOM5 sample ontology ${ }^{6}$ for primary cell and tissue samples, respectively, using indirect and direct 'is_a' and 'part_of' relationships. Ontology terms were manually selected to construct groups (facets) of samples that were mutually exclusive and to cover as broad histological and functional annotations as possible. 362 primary cell samples and 138 tissue and whole blood samples were grouped into 69 cell type facets and 41 organ/tissue facets, respectively (the groupings of samples into facets are provided in Supplementary Tables 10 and 11). A few samples were ignored because they were difficult to assign to a facet with certainty, which means that the number of samples within facets is slightly lower than the total number of samples.

For each facet, we defined a set of robustly expressed enhancers from the union of significantly expressed enhancers (see calculation of expression significance above) associated with each contained sample.

For motif search (see below), we identified the set of robust enhancers that were significantly deviating between facets using Kruskal-Wallis rank sum tests (BenjaminiHochberg $F D R<0.05$ ) and performed pair wise post-hoc tests (Nemenyi-Damico-WolfeDunn (NDWD) test ${ }^{59,60}$ using the R coin package ${ }^{61}$ to identify enhancers with significant differential expression (Bonferroni single-step adjusted $P<0.05$ ) between facets. Cell type facets and tissue/organ facets were analyzed separately. Each enhancer was considered differentially expressed in a facet with at least one pair-wise significant differential expression and overall positive standard linear statistics. This procedure means that we, for each robust enhancer, selected the facets, if any, with strong overall differential expression compared to all other facets. It should be noted that differential expression in this sense is not equivalent to facet-specific (exclusive) expression.

\section{Specificity and usage level analysis}

For each robust enhancer, we calculated a 'specificity' score across cell type and organ/ tissue facets. The specificity score was defined to range between 0 and 1 , where 0 means unspecific (ubiquitously expressed across facets) and 1 means specific (exclusively expressed in one facet).

In detail, specificity $(X)=1-\left(\right.$ entropy $\left.(X) / \log _{2}(N)\right)$, where $X$ is a vector of sample-average expression values for an enhancer over all facets (cell types and organs/tissues were 
analyzed separately) and $\mathrm{N}$ its cardinality (|X|, the number of facets). The same calculations were done for TPM and RLE normalized CAGE-derived expression levels of RefSeq protein-coding gene promoters (TSS +/- $500 \mathrm{bp}$ ).

In order to visualize the complexity and specialization of facets according to usage and specificity score of enhancers and genes, we counted the frequency of facet-used enhancers (significantly expressed in at least one contained sample) and gene promoters ( $\geq 1$ TPM in at least one sample) with a specificity score in any of 20 bins distributed between 0 and 1 . The number of robustly expressed enhancers and genes per sample were normalized to enhancers and genes per million mapped tags, utilizing the total number of mapped CAGE tags in each sample, and further log-transformed. The counts per million mapped tags were visualized in box plots split by facet (only facets with more than one contained sample were considered).

\section{Motif analysis on differentially specific enhancer sets}

To identify and compare motif signatures characterizing facet-specific enhancers (permissive set) we applied de novo motif analyses. Motif enrichment was analyzed using HOMER. Enhancer regions (400bp) were compared to $\sim 50,000$ randomly selected genomic fragments of the same region size, as described above. Twenty-five motifs were searched for a range of motif lengths (7-14 bp) resulting in a set of 200 de novo motifs per set, which was further filtered to remove redundant motifs. In parallel, we also used HOMER to calculate the enrichment of ChIP-Seq derived motifs. Motif collections including search parameters for all facets are deposited in the web database at http://enhancer.binf.ku.dk. Known transcription factor motifs were used to compare motif enrichment between facets.

\section{Hierarchical clustering of samples}

Tissue and primary cell samples mapped to ontology facets were clustered by complete linkage agglomerative hierarchical clustering based on Jensen-Shannon (JS) divergence ${ }^{59}$. In detail, expression values for all enhancers in the permissive set were normalized to sum to 1 for each sample and the square root (proper distance metric) of all pair wise JS divergences between samples was calculated. Manually selected clades of samples were analyzed for differential expression in a similar way as was done for facets (see above). In summary, differentially expressed enhancers (robust set) were identified by Kruskal-Wallis rank sum tests (Benjamini-Hochberg $F D R<0.05$ ) and subsequent NDWD post-hoc tests were performed to find all significant pair wise differences (Bonferroni single-step adjusted $P<0.05)$ between clades.

\section{Hierarchical clustering of enhancers}

We used matrices describing each enhancer expression in TPMs for each facet (primary cell facets and tissue facets were clustered independently) and clustered these by complete linkage agglomerative hierarchical clustering using Euclidan distances, as implemented in the gputools R package ${ }^{62}$, and ran these in parallel on a GTX960 Nvidia GPU. Due to limited memory in the GPU, we reduced the matrices to enhancers with total expression > $2.5 \mathrm{TPM}$ in the primary cell set and >0.6 TPM in the tissue/organ set, resulting in sets of roughly 22.500 enhancers each. To make sure these results were stable, we also explored 
normalization using fold change vs. background in each facet instead of TPM normalization, which resulted in very similar results (data not shown).

We then used the cutree method to select 5 sub-clusters in each tree, starting from the root. Enhancers in each set were then extended +-300 nt from their midpoints, and $\mathrm{CpG}$ islands and observed / expected $\mathrm{CpG}$ ratios were calculated. The resulting sub-clusters broke up enhancers into 201 and 247 ubiquitous enhancers (u-enhancers) defined by cell type and tissue facets, respectively, (these sets intersect by 106 enhancers) and non-ubiquitous enhancers. To summarize the features of u-enhancers in terms of expression width and variance, identified in a single plot, we used those enhancers falling into u-enhancer group from the tissue clustering. We then plotted the mean TPM over all tissue facets, as well as the coefficient of variation (expression variance over all tissue facets scaled by mean expression). Then we repeated this for the remaining enhancers (non-u-enhancers).

\section{Zebrafish reporter transgenesis experiments}

We selected enhancers for validation based on human-zebrafish conservation $(>70 \%$ sequence identity over $100 \mathrm{nt}$, hg19 vs DanRer7) in order to take into account the large evolutionary separation between the two species, and selected enhancers that were only expressed in a subset of tissues/cells. We did not take epigenetic data (ChIP/DHS etc.) into consideration. We also selected three negative control regions, chosen randomly from the human genome with the following constraints: low conservation with zebrafish and no other enhancer-selective feature, that is, no DNase hypersensitivity, no H3K4me1 or H3K27ac signals and CAGE signal only at noise levels.

Selected human enhancers (CRE1-5) were amplified from human genomic DNA using primers (Supplementary Table 9). PCR products were purified using NucleoSpin Gel and PCR Clean-up Kit (Macherey Nagel) and were digested using appropriate enzymes (listed in Supplementary Table 9). Human enhancers were cloned into EcoRV/SpeI or HindIII/EcoRI sites of pDB896 vector (gift from Darius Balciunas) upstream of zebrafish gata2 promoter ${ }^{63,64}$ and YFP reporter gene. Plasmid DNA was purified using NucleoBond® Xtra Midi Kit (Macherey Nagel) and quality checked by sequencing before injections.

Zebrafish stocks (Danio rerio) were kept and used according to Home Office regulations (UK) at the University of Birmingham. For these experiments wild-type fish (AB* strain) were used. Adults were crossed pairwise and eggs were collected 10-15 minutes after fertilization. Microinjection solutions contained $30 \mathrm{ng} / \mu \mathrm{l}$ of plasmid DNA, $0,2 \%$ of phenol red (Sigma) and $15 \mathrm{ng} / \mu \mathrm{l}$ of Tol2 mRNA transcribed in vitro from $\mathrm{pCS} 2$ :Tol2 plasmid using mMESSAGE machine SP6 Kit (Ambion). Injections were performed through the chorion and into the cytoplasm of zygotes using an analogue pressure-controlled microinjector (Tritech Research). More than 200 eggs were injected per construct and experiments were replicated at least three times. Embryos were kept according to ${ }^{65}$ in E3 Medium containing $50 \mathrm{ng} / \mathrm{ml}$ of gentamicin (Fisher Scientific) and 0,03\% phenylthiourea (PTU, Sigma) in an incubator at $28.5^{\circ} \mathrm{C}$.

Injected embryos were screened during the first 5 days post-fertilization using a Nikon SMZ1500 fluorescence stereomicroscope. Specific expression patterns were documented at 
$48 \mathrm{hpf}$ and levels of expression were quantified by counting the number of embryos showing enhancer-specific expression. In order to control for overall background activity from the construct (ie, promoter, backbone) an empty pDB896 vector containing gata2 zebrafish promoter linked to the reporter gene but lacking an enhancer sequence was used. Any tissuespecific enrichment shown by enhancer-containing vectors over the activity shown by the empty control vector was considered enhancer-specific. Additionally, three negative regions were also cloned to check the specificity of the enhancer selection process. These regions were chosen randomly from the human genome to have low conservation with zebrafish and no other enhancer-selective feature, that is, no DNase I hypersensitivity, no H3K4me1 or H3K27ac signals and CAGE signal only at noise levels. In parallel, 5 selected human enhancers were also analyzed. See Supplementary Table 9 for a summary of zebrafish validations, including expression patterns, signal strengths and primers.

\section{Analysis of cohesin data}

We used MCF7 cell ChIP experiments with antibodies targeting STAG1 and RAD21 proteins, downloaded from the Short Read archive (accession ERR011980, ERR011982). These were mapped using Bowtie ${ }^{50}$ with standard settings but discarding non-unique hits, and peak-called using MACS ${ }^{53}$ with default settings. We then used the intersection between peak sets as proxy binding sites for the cohesin complex.

\section{Linking TSSs and enhancers by expression correlations}

We identified all intra-chromosomal enhancer-promoter pairs (470,315 cases, permissive set of enhancers and unique locations of RefSeq protein-coding gene transcript TSSs $+/-500$ bp) within $500 \mathrm{~kb}$, in which the TSS was expressed >1 TPM in at least one sample, and performed Pearson correlation tests between the expression of such pairs: $64 \%$ of enhancers had at least one significant association (Benjamini-Hochberg $F D R<=1 \mathrm{e}-5$ ) within that distance. On average, a TSS was associated with 4.9 enhancers and an enhancer with 2.4 TSSs.

Next, we identified which predicted associations were supported by ENCODE ChIA-PET (via RNAPII (MMS-126R)) interaction data ${ }^{21}$ from four ENCODE cell lines (HCT-116, HeLa-S3, K562, MCF-7) by requiring an overlap of both enhancer and promoter in both (and different) sites of a ChIA-PET interaction pair. An association was considered supported if it overlapped in this way with any cell line replicate of interactions.

For comparison, the fraction of 1,672,958 published ${ }^{10}$ predicted enhancer-promoter associations derived from DNase data supported by ENCODE ChIA-PET interaction data was calculated.

\section{Analysis of genomic clusters of densely positioned enhancers}

By pairwise distance calculations between CAGE enhancers, we identified clusters of densely positioned enhancers in the genome. 815 regions of length $>=2 \mathrm{~kb}$ containing $>2$ enhancers were identified. Of these, 198 regions contained enhancers whose average pairwise expression correlation (Pearson's $r$ ) were $>=0.75$. The expression of associated Refseq genes (see 'Linking TSSs and enhancers by expression correlations') as well as their 
enrichment of gene ontology biological process terms (via the DAVID tool ${ }^{66}$ ) were compared to that of genes associated with non-clustered enhancers.

\section{Inferring regulatory architectures by multiple linear regression}

Multiple linear regression was performed for all 25,144 expressed (max TPM >1) RefSeq TSSs with at least ten FANTOM5 CAGE-defined enhancers within $500 \mathrm{~kb}$. Enhancers were ranked by proximity to the TSS and the expression values across all samples of the ten closest were used as predictor variables in a model with the TSS expression as response variable. The expression data of enhancers and TSSs were centered and rescaled. 2,206 TSS models, considering in total 11,386 enhancers, with $R^{2} \geq 0.5$ were considered for further analyses. We also fitted a simple linear regression model using each enhancer as predictor variable on their own, in order to compare the predictive power of a single enhancer to the power of using all ten. We defined a new measure of 'proportional contribution' to the variance explained as the ratio between simple linear regression $r^{2}$ and multiple linear regression $R^{2}$, for each enhancer among the ten considered for each TSS. This measure yielded highly similar ranking results of enhancers as the $R^{2}$ contribution averaged over orderings among regressors ${ }^{67,68}$ and $R^{2}$ decorrelation decomposition ${ }^{67,69}$ (data not shown), implemented in the 'relaimpo' $\mathrm{R}$ package ${ }^{37,69}$ (lmg and car methods, respectively). We used ranking of enhancers according to proportional contribution and within-model enhancerenhancer correlations to identify TSSs with different enhancer architectures. Redundant enhancers were identified for TSSs that had enhancers that were, by proportional contribution, ranked second and onwards with at least some proportional contribution $(>0.2)$ and high correlation (Pearson's $r>0.7$ ) with any other of the nine enhancers in the model. Patterning architectures were considered for enhancers in non-redundant models that were, by proportional contribution, ranked second and onwards with at least some proportional contribution $(>0.2)$ and low correlation (Pearson's $r<0.3$ ) with all other of the nine enhancers in the model. Penalized lasso-based regression was used to reduce the number of enhancers in the models. The optimal models were selected using 100-fold cross validation and the largest value of lambda such that the mean squared error was within one standard error of the minimum, using the $\mathrm{R}$ package glmnet $^{29,37}$

\section{SNP analysis}

The NIH NHGRI catalog of published genome-wide association studies ${ }^{29}$ (GWAS catalog, downloaded May 7, 2012) contained 7,899 SNP-disease/trait associations. We extended this set to 190,356 autosomal associations by propagating disease/trait associations to proxy SNPs using the SNAP proxy search tool ${ }^{70}$ (http://www.broadinstitute.org/mpg/snap/) based on linkage disequilibrium $\left(r^{2}>0.8\right)$ between SNPs (within $250 \mathrm{~kb}$ ) in any of the three populations in the 1000 genomes project pilot $^{71}$ data. The 1000 genome data coordinates were in hg18 coordinates and were mapped to hg19 using the UCSC liftOver tool ${ }^{72}$.

For robust enhancers (center $+/-200 \mathrm{bp}$ ), promoters (unique locations of RefSeq proteincoding gene transcript TSSs $+/-200 \mathrm{bp}$ ), exons (unique locations of RefSeq protein-coding gene transcript inner exons), and random regions (described above), we calculated the number of overlapping and non-overlapping GWAS SNPs associated with each disease/trait in the extended GWAS catalog. Non-associated SNPs were extracted from the NCBI single 
nucleotide polymorphism database (dbSNP, build 135). For each genomic feature and disease/trait with an odds ratio $>1$, we tested whether the observed overlap was significantly greater than expected (Fisher's exact test $P<0.01$ ). Only diseases/traits with more than three SNPs overlapping were tested. The same analysis was repeated for each set of significantly expressed enhancers associated with each facet. For ease of visualization and interpretation, only odds ratios for which the filtering criteria on both significance and overlap number were met are shown. Lists of enhancer-overlapped GWAS SNPs are in S16.

\section{Statistical tests, visualization and tools used}

Statistical tests were done in the R environment (http://www.R-project.org). Graphs were made using lattice, ggplot 2 and gplots $\mathrm{R}$ packages. Cluster trees were generated by the $\mathrm{APE}^{73} \mathrm{R}$ package and visualized using the FigTree software (http://tree.bio.ed.ac.uk/ software/figtree/). Intersections of and distances between various genomic features were calculated using BEDTools ${ }^{74}$

\section{Supplementary Material}

Refer to Web version on PubMed Central for supplementary material.

\section{Acknowledgements}

FANTOM5 was made possible by a Research Grant for RIKEN Omics Science Center from MEXT to YH and a Grant of the Innovative Cell Biology by Innovative Technology (Cell Innovation Program) from the MEXT, Japan to YH. The AS group was supported by funds from the European Research Council FP7/2007-2013/ERC \#204135, the Novo Nordisk and Lundbeck foundations. Work in MRs group was funded by grants from the Deutsche Forschungsgemeinschaft (RE 1310/7, 11, 13) and Rudolf Bartling Stiftung. FM and IME were supported by "BOLD" Marie Curie ITN and "ZF-Health" Integrated project of the European Commission. We thank i) Shohei Noma, Mizuho Sakai, and Hiroshi Tarui for RNA-seq and sRNA-seq preparation, ii) RIKEN GeNAS for generation and sequencing of the Heliscope CAGE libraries, Illumina RNAseq and sRNAseq, iii) the Copenhagen National High-throughput DNA Sequencing Center for Illumina CAGE-seq, iv) Anders Albrechtsen, Ida Moltke, Wyeth Wasserman for advice, and v) the Netherlands Brain Bank for post-mortem human brain material.

\section{REFERENCES}

1. Bulger M, Groudine M. Enhancers: The abundance and function of regulatory sequences beyond promoters. Developmental Biology. 2010; 339:250-257. [PubMed: 20025863]

2. Lenhard B, Sandelin A, Carninci P. Metazoan promoters: emerging characteristics and insights into transcriptional regulation. Nat Rev Genet. 2012; 13:233-245. [PubMed: 22392219]

3. Banerji J, Rusconi S, Schaffner W. Expression of a $\beta$-globin gene is enhanced by remote SV40 DNA sequences. Cell. 1981; 27:299-308. [PubMed: 6277502]

4. Kim T-K, et al. Widespread transcription at neuronal activity-regulated enhancers. Nature. 2010; 465:182-187. [PubMed: 20393465]

5. Kodzius R, et al. CAGE: cap analysis of gene expression. Nature methods. 2006; 3:211-222. [PubMed: 16489339]

6. The FANTOM Consortium. A promoter level mammalian expression atlas.. Submitted

7. The ENCODE Consortium. P. An integrated encyclopedia of DNA elements in the human genome. Nature. 2012; 489:57-74. [PubMed: 22955616]

8. Kheradpour P, et al. Systematic dissection of regulatory motifs in 2000 predicted human enhancers using a massively parallel reporter assay. Genome Research. 2013; 23:800-811. [PubMed: 23512712]

9. Fort, A., et al. Deep transcriptome profiling reveals that retrotransposons regulate pluripotency.. Submitted 
10. Thurman RE, et al. The accessible chromatin landscape of the human genome. Nature. 2012; 489:75-82. [PubMed: 22955617]

11. Ntini E, et al. Polyadenylation site-induced decay of upstream transcripts enforces promoter directionality. Nat Struct Mol Biol. 2013; 20:923-928. [PubMed: 23851456]

12. Almada AE, Wu X, Kriz AJ, Burge CB, Sharp PA. Promoter directionality is controlled by U1 snRNP and polyadenylation signals. Nature. 2013; 499:360-363. [PubMed: 23792564]

13. Djebali S, et al. Landscape of transcription in human cells. Nature. 2012; 489:101-108. [PubMed: 22955620]

14. Kowalczyk MS, et al. Intragenic Enhancers Act as Alternative Promoters. Molecular Cell. 2012; 45:447-458. [PubMed: 22264824]

15. Valen E, et al. Biogenic mechanisms and utilization of small RNAs derived from human proteincoding genes. Nat Struct Mol Biol. 2011; 18:1075-1082. [PubMed: 21822281]

16. Taft RJ, et al. Tiny RNAs associated with transcription start sites in animals. Nat Genet. 2009; 41:572-578. [PubMed: 19377478]

17. Core LJ, Waterfall JJ, Lis JT. Nascent RNA Sequencing Reveals Widespread Pausing and Divergent Initiation at Human Promoters. Science. 2008; 322:1845-1848. [PubMed: 19056941]

18. Rönnerblad, M., et al. Analysis of the DNA methylome and transcriptome in granulopoiesis reveal timed changes and dynamic enhancer methylation.. Submitted

19. Biddie SC, et al. Transcription Factor AP1 Potentiates Chromatin Accessibility and Glucocorticoid Receptor Binding. Molecular Cell. 2011; 43:145-155. [PubMed: 21726817]

20. Schmidt D, et al. A CTCF-independent role for cohesin in tissue-specific transcription. Genome Res. 2010; 20:578-588. [PubMed: 20219941]

21. Li G, et al. Extensive Promoter-Centered Chromatin Interactions Provide a Topological Basis for Transcription Regulation. Cell. 2012; 148:84-98. [PubMed: 22265404]

22. Chepelev I, Wei G, Wangsa D, Tang Q, Zhao K. Characterization of genome-wide enhancerpromoter interactions reveals co-expression of interacting genes and modes of higher order chromatin organization. Cell Res. 2012; 22:490-503. [PubMed: 22270183]

23. Fraser P, Pruzina S, Antoniou M, Grosveld F. Each hypersensitive site of the human beta-globin locus control region confers a different developmental pattern of expression on the globin genes. Genes \& Development. 1993; 7:106-113. [PubMed: 8422981]

24. Dostie J, et al. Chromosome Conformation Capture Carbon Copy (5C): A massively parallel solution for mapping interactions between genomic elements. Genome Research. 2006; 16:12991309. [PubMed: 16954542]

25. Barolo S. Shadow enhancers: Frequently asked questions about distributed cis-regulatory information and enhancer redundancy. Bioessays. 2011; 34:135-141. [PubMed: 22083793]

26. Schaffner G, Schirm S, Müller-Baden B, Weber F, Schaffner W. Redundancy of information in enhancers as a principle of mammalian transcription control. J. Mol. Biol. 1988; 201:81-90. [PubMed: 2843647]

27. Whyte WA, et al. Master Transcription Factors and Mediator Establish Super-Enhancers at Key Cell Identity Genes. Cell. 2013; 153:307-319. [PubMed: 23582322]

28. Göring HHH, et al. Discovery of expression QTLs using large-scale transcriptional profiling in human lymphocytes. Nat Genet. 2007; 39:1208-1216. [PubMed: 17873875]

29. Hindorff LA, et al. Potential etiologic and functional implications of genome-wide association loci for human diseases and traits. Proceedings of the National Academy of Sciences. 2009; 106:93629367.

30. Ward LD, Kellis M. HaploReg: a resource for exploring chromatin states, conservation, and regulatory motif alterations within sets of genetically linked variants. Nucleic Acids Research. 2011; 40:D930-D934. [PubMed: 22064851]

31. Maurano MT, Wang H, Kutyavin T, Stamatoyannopoulos JA. Widespread Site-Dependent Buffering of Human Regulatory Polymorphism. PLoS Genet. 2012; 8:e1002599. [PubMed: 22457641] 
32. Mercer EM, et al. Multilineage Priming of Enhancer Repertoires Precedes Commitment to the B and Myeloid Cell Lineages in Hematopoietic Progenitors. Immunity. 2011; 35:413-425. [PubMed: 21903424]

33. Ostuni R, et al. Latent Enhancers Activated by Stimulation in Differentiated Cells. Cell. 2013; 152:157-171. [PubMed: 23332752]

34. Rada-Iglesias A, et al. A unique chromatin signature uncovers early developmental enhancers in humans. Nature. 2010; 470:279-283. [PubMed: 21160473]

35. Shen Y, et al. A map of the cis-regulatory sequences in the mouse genome. Nature. 2012; 488:116120. [PubMed: 22763441]

36. Heinz S, et al. Simple Combinations of Lineage-Determining Transcription Factors Prime cisRegulatory Elements Required for Macrophage and B Cell Identities. Molecular Cell. 2010; 38:576-589. [PubMed: 20513432]

37. Friedman J, Hastie T, Tibshirani R. Regularization Paths for Generalized Linear Models via Coordinate Descent. Journal of Statistical Software. 2010; 33:1-22. [PubMed: 20808728]

38. Gehrig J, et al. Automated high-throughput mapping of promoter-enhancer interactions in zebrafish embryos. Nature methods. 2009; 6:911-916. [PubMed: 19898487]

39. Kanamori-Katayama M, et al. Unamplified cap analysis of gene expression on a single-molecule sequencer. Genome Research. 2011; 21:1150-1159. [PubMed: 21596820]

40. Khalil AM, et al. Many human large intergenic noncoding RNAs associate with chromatinmodifying complexes and affect gene expression. Proceedings of the National Academy of Sciences. 2009; 106:11667-11672.

41. Robinson MD, McCarthy DJ, Smyth GK. edgeR: a Bioconductor package for differential expression analysis of digital gene expression data. Bioinformatics. 2009; 26:139-140. [PubMed: 19910308]

42. Hoffman MM, et al. Unsupervised pattern discovery in human chromatin structure through genomic segmentation. Nature methods. 2012; 9:473-476. [PubMed: 22426492]

43. Ernst J, Kellis M. ChromHMM: automating chromatin-state discovery and characterization. Nature methods. 2012; 9:215-216. [PubMed: 22373907]

44. Heintzman ND, et al. Histone modifications at human enhancers reflect global cell-type-specific gene expression. Nature. 2009; 459:108-112. [PubMed: 19295514]

45. Marshall OJ. PerlPrimer: cross-platform, graphical primer design for standard, bisulphite and realtime PCR. Bioinformatics. 2004; 20:2471-2472. [PubMed: 15073005]

46. Trapnell C, Pachter L, Salzberg SL. TopHat: discovering splice junctions with RNA-Seq. Bioinformatics. 2009; 25:1105-1111. [PubMed: 19289445]

47. Trapnell C, et al. Transcript assembly and quantification by RNA-Seq reveals unannotated transcripts and isoform switching during cell differentiation. Nat Biotechnol. 2010; 28:511-515. [PubMed: 20436464]

48. Preker P, et al. RNA Exosome Depletion Reveals Transcription Upstream of Active Human Promoters. Science. 2008; 322:1851-1854. [PubMed: 19056938]

49. Takahashi H, Lassmann T, Murata M, Carninci P. 5' end-centered expression profiling using capanalysis gene expression and next-generation sequencing. Nat Protoc. 2012; 7:542-561. [PubMed: 22362160]

50. Langmead B, Trapnell C, Pop M, Salzberg SL. Ultrafast and memory-efficient alignment of short DNA sequences to the human genome. Genome Biol. 2009; 10:R25. [PubMed: 19261174]

51. Carninci P, et al. Genome-wide analysis of mammalian promoter architecture and evolution. Nat Genet. 2006; 38:626-635. [PubMed: 16645617]

52. Pham TH, et al. Dynamic epigenetic enhancer signatures reveal key transcription factors associated with monocytic differentiation states. Blood. 2012; 119:e161-e171. [PubMed: 22550342]

53. Zhang Y, et al. Model-based Analysis of ChIP-Seq (MACS). Genome Biol. 2008; 9:R137. [PubMed: 18798982]

54. Schmidl C, et al. Lineage-specific DNA methylation in T cells correlates with histone methylation and enhancer activity. Genome Research. 2009; 19:1165-1174. [PubMed: 19494038] 
55. Klug M, Rehli M. Functional analysis of promoter CpG methylation using a CpG-free luciferase reporter vector. Epigenetics. 2006; 1:127-130. [PubMed: 17965610]

56. Rehli M. PU.1 and Interferon Consensus Sequence-binding Protein Regulate the Myeloid Expression of the Human Toll-like Receptor 4 Gene. Journal of Biological Chemistry. 2000; 275:9773-9781. [PubMed: 10734131]

57. Li LC, Dahiya R. MethPrimer: designing primers for methylation PCRs. Bioinformatics. 2002; 18:1427-1431. [PubMed: 12424112]

58. Ehrich M, et al. Quantitative high-throughput analysis of DNA methylation patterns by basespecific cleavage and mass spectrometry. Proceedings of the National Academy of Sciences. 2005; 102:15785-15790.

59. Lin J. Divergence measures based on the Shannon entropy. IEEE Trans. Inform. Theory. 1991; 37:145-151.

60. Nonparametric statistical methods. Wiley-Interscience; 1999.

61. Hothorn T, Hornik K, Van De Wiel MA, Zeileis A. A lego system for conditional inference. The American Statistician. 2006; 60:257-263.

62. Buckner J, et al. The gputools package enables GPU computing in R. Bioinformatics. 2009; 26:134-135. [PubMed: 19850754]

63. Ellingsen S, et al. Large-scale enhancer detection in the zebrafish genome. Development. 2005; 132:3799-3811. [PubMed: 16049110]

64. Meng A, Tang H, Ong BA, Farrell MJ, Lin S. Promoter analysis in living zebrafish embryos identifies a cis-acting motif required for neuronal expression of GATA-2. Proc. Natl. Acad. Sci. U.S.A. 1997; 94:6267-6272. [PubMed: 9177206]

65. The Zebrafish Book. A Guide for the Laboratory Use of Zebrafish(Danio rerio). University of Oregon Press; 1995.

66. Huang DW, Sherman BT, Lempicki RA. Systematic and integrative analysis of large gene lists using DAVID bioinformatics resources. Nat Protoc. 2008; 4:44-57.

67. Zuber V, Strimmer K. High-Dimensional Regression and Variable Selection Using CAR Scores. Statistical Applications in Genetics and Molecular Biology. 10:1-27.

68. Chevan A, Sutherland M. Hierarchical Partitioning. The American Statistician. 1991; 45:90-96.

69. Groemping U. Relative Importance for Linear Regression in R: The Package relaimpo. Journal of Statistical Software. 2006; 17:27.

70. Johnson AD, et al. SNAP: a web-based tool for identification and annotation of proxy SNPs using HapMap. Bioinformatics. 2008; 24:2938-2939. [PubMed: 18974171]

71. Durbin RM, et al. A map of human genome variation from population-scale sequencing. Nature. 2010; 467:1061-1073. [PubMed: 20981092]

72. Rhead B, et al. The UCSC Genome Browser database: update 2010. Nucleic Acids Research. 2009; 38:D613-D619. [PubMed: 19906737]

73. Paradis E, Claude J, Strimmer K. APE: Analyses of Phylogenetics and Evolution in R language. Bioinformatics. 2004; 20:289-290. [PubMed: 14734327]

74. Quinlan AR, Hall IM. BEDTools: a flexible suite of utilities for comparing genomic features. Bioinformatics. 2010; 26:841-842. [PubMed: 20110278] 

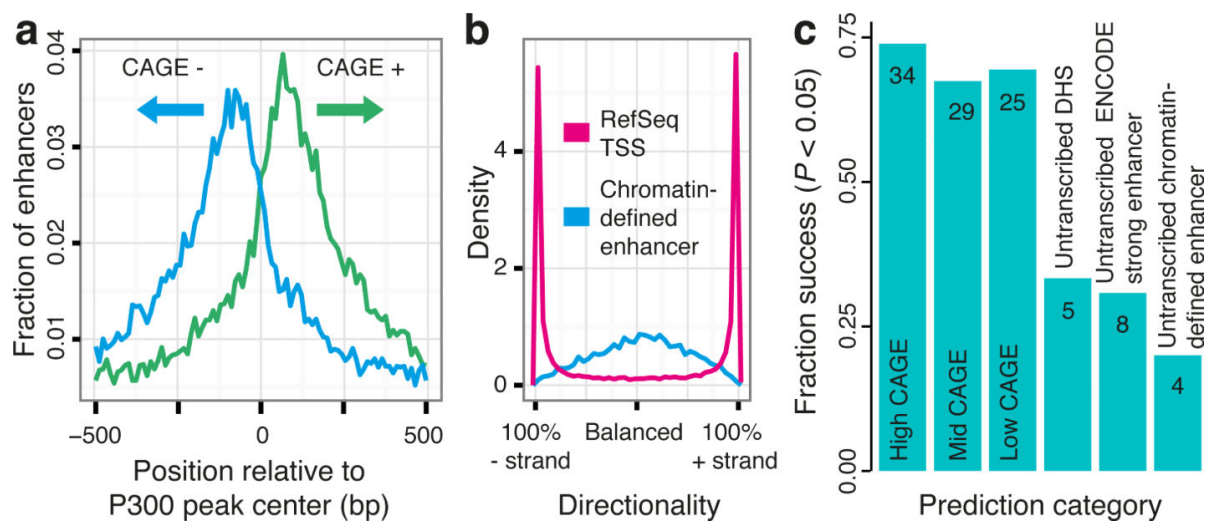

Figure 1. Bidirectional capped RNAs is a signature feature of active enhancers

a, Enhancers identified by co-occurrence of H3K27ac and H3K4me1 ChIP-seq data ${ }^{7}$, centered on P300 binding sites, in HeLa cells were overlaid with HeLa CAGE data (unique positions of CAGE tag 5 ' ends, smoothed by a 5 bp window), revealing a bidirectional transcription pattern. Horizontal axis shows the $\pm 500 \mathrm{bp}$ region around enhancer midpoints. b, Density plot illustrating the difference in directionality of transcription according to FANTOM5 pooled CAGE tags mapped within \pm 300 bp of 22,486 TSSs of RefSeq proteincoding genes and center positions of 10,138 HeLa enhancers defined as above.

c, Success rates of in vitro enhancer assays in HeLa cells. Vertical axis shows the fraction of active enhancers (success defined by Student's t-test, $P<0.05 \mathrm{vs}$. random regions; also see Supplementary Figure 9). Numbers of successful assays are shown on the respective bar. See main text for details. 

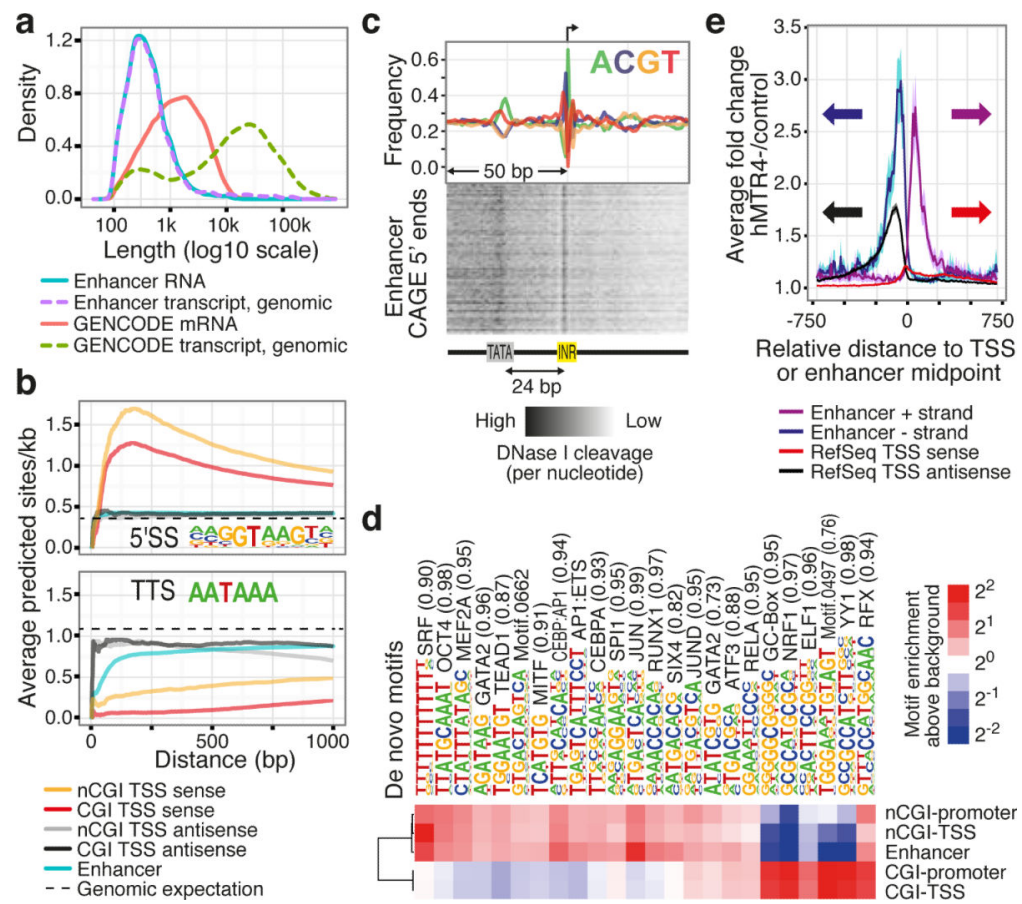

Figure 2. Features distinguishing enhancer TSSs from mRNA TSSs

a, Densities of the genomic and processed RNA lengths of transcripts starting from enhancer TSSs and mRNA TSSs using assembled RNA-seq reads from 13 pooled FANTOM5 libraries.

b, Frequencies of RNA processing motifs (5' splice motif (5'sS, left panel) and the transcription termination site hexamer (TTS, right panel)) around enhancer and mRNA TSSs. Vertical axis shows the average number of predicted sites per bp within a certain window size from the TSS (horizontal axis) in which the motif search was done. Dashed lines indicate expected hit density from random genomic background. The window always starts at the gene or enhancer CAGE summits and expands in the sense direction.

c, Average nucleotide frequencies (top panel) and DNase I cleavage patterns (lower panel) of enhancer CAGE peaks (arrow at +1 indicates position of the main enhancer CAGE peaks; direction of transcription goes left to right) reveal distinct cleavage patterns at sequences resembling the INR and TATA elements.

d, De novo motif enrichment analyses around enhancers and non-enhancer FANTOM5 CAGE-defined TSSs (CAGE TSSs matching annotated TSSs are referred to as "promoters"), contingent on CGI overlap. Top enriched/depleted motifs are shown along with their best-known motif match name. Enrichment vs. random background is presented as a heat map.

e, Vertical axis shows average HeLa CAGE expression fold change vs. control at enhancers and RefSeq TSSs after exosome depletion. Horizontal axis shows position relative to the TSS or the center of the enhancer. Translucent colors indicate the $95 \%$ confidence interval of the mean. 

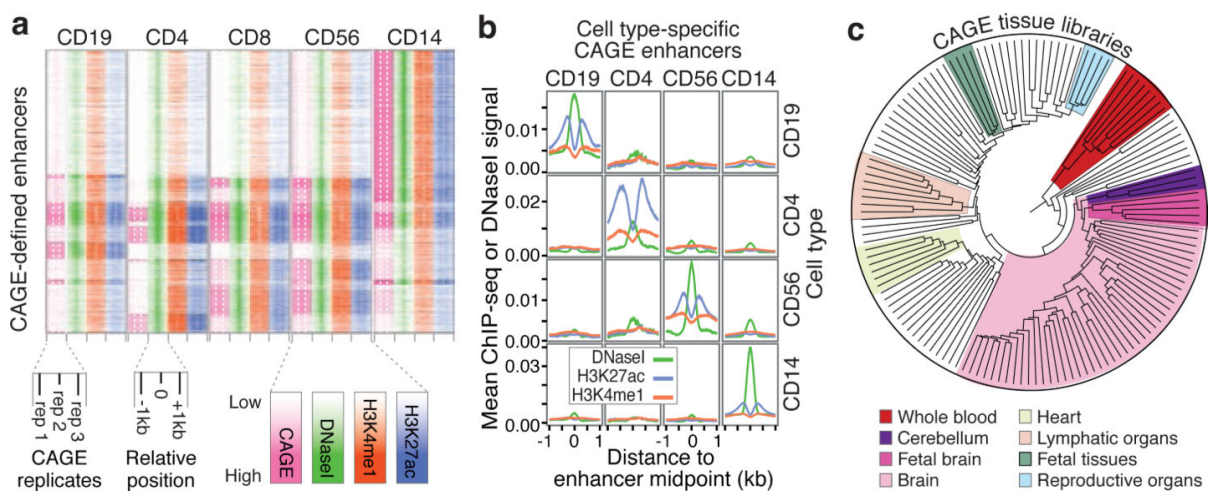

Figure 3. CAGE expression identifies cell type-specific enhancer usage

a, Relationship between CAGE and histone modifications in blood cells. Rows represent CAGE-defined enhancers that are ordered based on hierarchical clustering of CAGE expression. Columns for the CAGE tags (pink) represent the expression intensity for three biological replicates. DNase I hypersensitivity and H3K27ac and H3K4me1 ChIP-seq signals $\pm 1 \mathrm{~kb}$ around the enhancer midpoints are shown in green, blue and orange, respectively.

b, Mean signal of DNase-seq as well as ChiP-seq for H3K27ac and H3K4me1 (vertical axis) per cell type (rows) in $\pm 1 \mathrm{~kb}$ regions (horizontal axis) around enhancer midpoints, for enhancers with blood cell type-specific CAGE expression (columns).

c, Dendrogram resulting from agglomerative hierarchical clustering of tissue samples based on their enhancer expression: each leaf of the tree represents one CAGE tissue sample (for a labeled tree and the corresponding results on primary cell samples, see Supplementary Figs 18 and 19). Sub-trees dominated by one tissue/organ type or morphology are highlighted.

Some of the enhancers responsible for the fetal-specific subgroup in the larger brain subtree are validated in vivo (Fig. 4). 

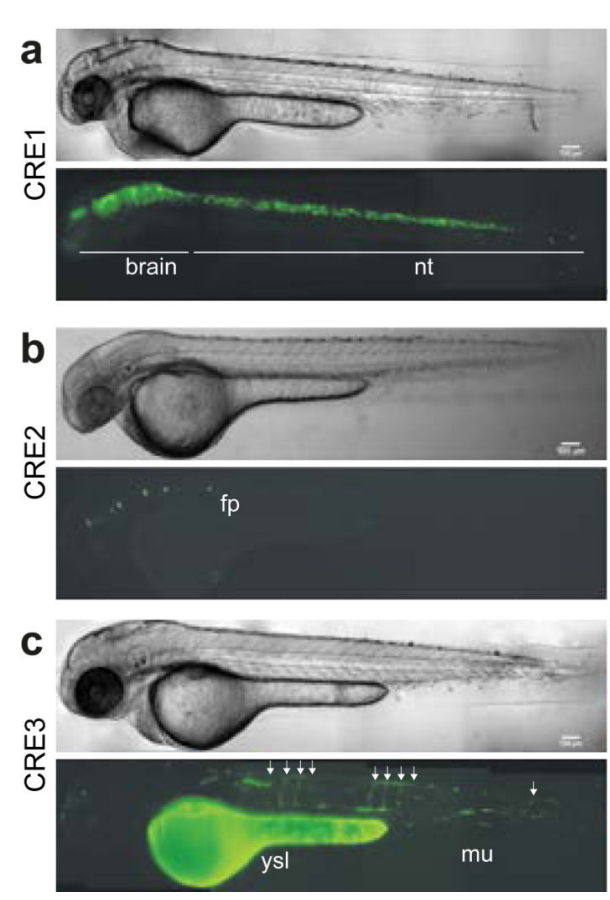
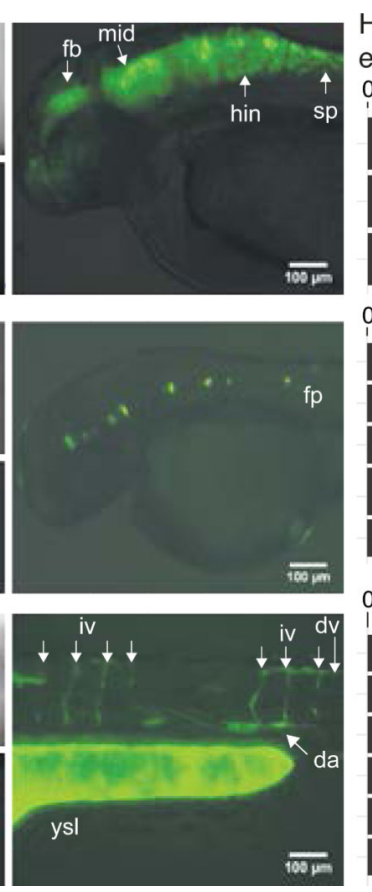

Human enhancer expression (TPM)

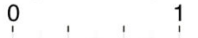

Neuron

Astrocyte

Brain

$0,1,3$ Neural stem cell

Neuron Astrocyte Spinal cord Brain

\begin{tabular}{|c|c|}
\hline | | | | | \\
\hline Salivary gland \\
\hline Blood vessel of \\
epithelial cell \\
Endothelial cell of \\
hepatic sinusoid \\
Kidney \\
epithelial cell \\
Urothelial cell \\
Respiratory \\
epithelial cell
\end{tabular}

Figure 4. In vivo validation in zebrafish of tissue-specific enhancers

Validations of in vivo activity of CAGE-defined human enhancers CRE1-3 in zebrafish embryos at long-pec stage. Each panel shows, from left to right: i) representative YFP and brightfield images of embryos injected with the human enhancer gata2 promoter reporter gene construct. Muscle (mu) and yolk syncytial layer (ysl) activities are background expression coming from the gata2 promoter-containing reporter construct. All images are lateral, head to the left. ii) YFP zoom-ins and iii) CAGE expression in TPM in human tissues/cell types for the enhancer. Note the correspondence between zebrafish and human enhancer usage/expression. Supplementary Figure 20 shows UCSC browser images of each selected enhancer.

a, CRE1, 230kb upstream of the MEFC2 gene, drives highly robust expression in the brain (brain) and neural tube (nt). Right panel gives zoom-in overlay image showing expression in the forebrain (fb), midbrain (mid), hindbrain (hin) and spinal cord (sp).

b, CRE2, 5kb upstream of the POU3F2 gene, is active in the floor plate (fp). c, CRE3, 10kb upstream of the SOX7 gene TSS, shows specific expression in the vasculature (including intersegmental vessels (iv), dorsal vein (dv) and dorsal aorta (da)). 


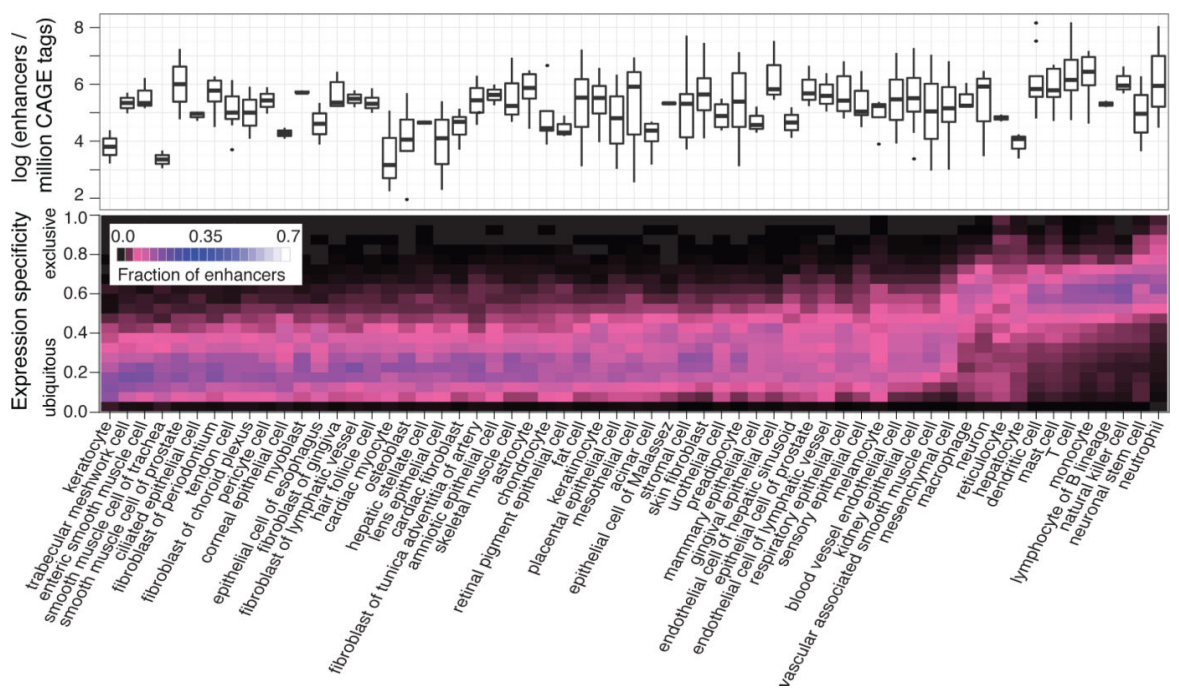

Figure 5. Enhancer usage and specificity in groups of cells

The upper panel gives the number of detected enhancers per million CAGE tags within each group (facet) of related cell type libraries. The expression specificity of the enhancers is shown as a heat map in the panel below. Colors show the fraction of expressed enhancers in each facet (columns) that are in each specificity range (rows). For corresponding plots on organ/tissue facets and genes, see Supplementary Figure 21. 

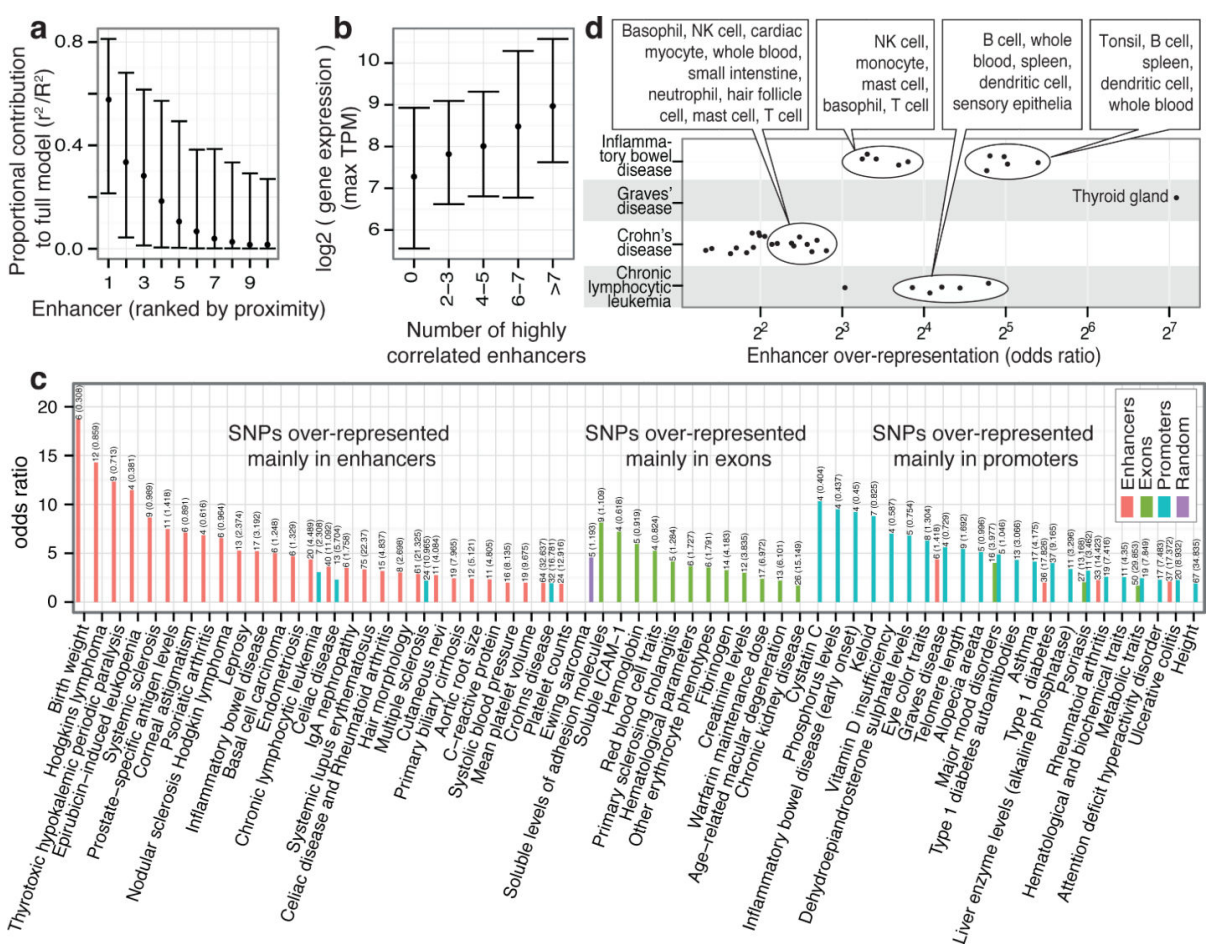

Figure 6. Linking enhancers to TSSs and disease-associated SNPs

a, The proportional contribution (See Methods) of the 10 most proximal enhancers within $500 \mathrm{~kb}$ of a TSS in a model explaining gene expression variance (vertical axis) as a function of enhancer expression. $\mathrm{X}$ axis indicates the position of the enhancer relative to the TSS: 1 the closest, etc. Bars indicate interquartile ranges and dots medians.

b, Relationship between the number of highly correlated ('redundant') enhancers per locus (horizontal axis) and the maximal expression (TPM) of the associated TSS in the same model over all CAGE libraries (vertical axis).

c, GWAS SNP sets preferentially overrepresented within enhancers, exons and mRNA promoters. The horizontal axis gives enrichment odds ratios. The vertical axis shows GWAS traits or diseases.

d, Diseases with GWAS associated SNPs over-represented in enhancers of certain expression facets. The horizontal axis gives the odds ratio as in panel $\mathrm{C}$, broken up by expression facets: each point represents the odds ratio of GWAS SNP enrichment for a disease (vertical axis) in a specific expression facet. Summary annotations of point clouds are shown. Also see Supplementary Figure 31. 